\title{
RESONANT SOLUTIONS OF THE PERIODICALLY FORCED KdV EQUATION
}

\author{
MARK BLENKINSOP, B.Math.
}

A thesis submitted to the Faculty of Graduate Studies and Research in partial fulfillment of the requirements for the degree of Master of Science

School of Mathematics and Statistics

Ottawa-Carleton Institute of Mathematics and Statistics

Carleton University

Ottawa, Ontario, Canada

April 2006

(C) Mark Blenkinsop, 2006 


$\begin{array}{ll}\begin{array}{l}\text { Library and } \\ \text { Archives Canada }\end{array} & \begin{array}{l}\text { Bibliothèque et } \\ \text { Archives Canada }\end{array} \\ \begin{array}{l}\text { Published Heritage } \\ \text { Branch }\end{array} & \begin{array}{l}\text { Direction du } \\ \text { Patrimoine de l'édition }\end{array} \\ \begin{array}{l}\text { 395 Wellington Street } \\ \text { Ottawa ON K1A 0N4 }\end{array} & \begin{array}{l}\text { 395, rue Wellington } \\ \text { Ottana ON K1A ON4 } \\ \text { Canada Oa }\end{array}\end{array}$

Your file Votre référence ISBN: 978-0-494-16491-4 Our file Notre référence ISBN: $978-0-494-16491-4$

NOTICE:

The author has granted a nonexclusive license allowing Library and Archives Canada to reproduce, publish, archive, preserve, conserve, communicate to the public by telecommunication or on the Internet, loan, distribute and sell theses worldwide, for commercial or noncommercial purposes, in microform, paper, electronic and/or any other formats.

The author retains copyright ownership and moral rights in this thesis. Neither the thesis nor substantial extracts from it may be printed or otherwise reproduced without the author's permission.
AVIS:

L'auteur a accordé une licence non exclusive permettant à la Bibliothèque et Archives Canada de reproduire, publier, archiver, sauvegarder, conserver, transmettre au public par télécommunication ou par l'Internet, prêter, distribuer et vendre des thèses partout dans le monde, à des fins commerciales ou autres, sur support microforme, papier, électronique et/ou autres formats.

L'auteur conserve la propriété du droit d'auteur et des droits moraux qui protège cette thèse. $\mathrm{Ni}$ la thèse ni des extraits substantiels de celle-ci ne doivent être imprimés ou autrement reproduits sans son autorisation.
In compliance with the Canadian

Privacy Act some supporting forms may have been removed from this thesis.

While these forms may be included in the document page count, their removal does not represent any loss of content from the thesis.
Conformément à la loi canadienne sur la protection de la vie privée, quelques formulaires secondaires ont été enlevés de cette thèse.

Bien que ces formulaires aient inclus dans la pagination, il n'y aura aucun contenu manquant. 


\section{Abstract}

This thesis examines the steady state solutions for the periodically forced Korteweg de Vries (fKdv) equation with general forcing. The solutions of the fKdV equation are not well known, and their study has been primarily on problems in which the forcing is a single sinusoidal mode. This research focuses on adapting pre-existing results for this topic to a general forcing in an attempt to broaden the understanding of the solutions for the generally forced $\mathrm{KdV}$ equation.

Using singular perturbation methods and multiple scale techniques, general matched, uniformly valid, leading order approximations are determined for the damped and undamped forced KdV equation. Following this, variation of the detuning parameter is examined, more specifically, analytical determination of peak formation and movement, as the solution is continuously varied.

Numerical solutions are used to verify results. First, undamped and damped leading order approximations are shown to correspond well to the numerical results. Next, the variation of the detuning parameter is investigated and leads to parametric continuations through a rich array of steady solutions. These qualitatively distinct non-dispersive solutions give rise to issues of peak overcrowding, solution domain saturation, and simultaneous multiple peak formation.

Examination of regions of stability shows that it is classified by peak location in the solution, in relation to the extrema of the non-dispersive solution, 
and extends the known stability characteristics for the single mode case. Regions of solution stability are dependent on stability within each layer, yielding ranges of the detuning parameter which correspond to either stable or unstable solutions. 


\section{Acknowledgments}

I would like to first and foremost thank my supervisor, Dr. David Amundsen, for all his help and efforts on my behalf. His tolerance and patience with me made it possible to complete this thesis, and my degree.

I would also like to thank my father, Dr. John Blenkinsop, for his advice and experience he shared with me both in the context of this thesis, and in relation to my degree.

Finally I would like to thank the rest of my family, friends, swimming coaches, and team mates for their support of my studies, though it sometimes consisted of ironic and sarcastic comment, and in particular my older brother, Adrian, for letting me get my Master's degree before him. 


\section{Contents}

1 Introduction 1

2 Analytic Approximations 5

2.1 Undamped Case . . . . . . . . . . . . 6

2.2 Damped Case . . . . . . . . . . . . . . 14

2.3 Peak Formation and Movement ............ 18

3 Numerical Examples $\quad 23$

3.1 Comparison of Analytic Approximations and Numerical Solutions 23

3.2 Parametric Continuation . . . . . . . . . . 29

3.2.1 Non-Dispersive Solutions with a Single Maximum and Minimum ................... 30

3.2.2 Non-Dispersive Solutions with Multiple Extrema and One Global Minimum . . . . . . . . . . 33

3.2.3 Non-Dispersive Solutions with Multiple Extrema and Multiple Global Minima . . . . . . . . . . . . 38

4 Stability of Steady Solutions $\quad 40$ 
4.1 Regions of Stability for the General Non-Dispersive Solution

4.2 Solution Stability for the Single Mode Forcing . . . . . . . . . 42

4.3 Solution Stability for the General Forcing . . . . . . . . . . . 43

5 Conclusion 45 


\section{List of Figures}

1 partial finger diagram illustrating known behaviour in the resonant band ........................... 19

2 non-dispersive solution derived from sample single mode forcing 20

3 solutions corresponding to each of the four points on the partial bifurcation diagram, Figure $1 \ldots \ldots 21$

4 forcing $f(x)=\frac{1}{10} \sin \left(\frac{x \pi}{2}\right)+\frac{1}{3} \sin (x \pi+1)$, with roots $\left(\alpha_{1}, \alpha_{2}, \alpha_{3}, \alpha_{4}\right)=$ $(0.7724,1.6288,2.6034,3.7221) \ldots \ldots \ldots 25$

5 approximate solution for $f(x)=\frac{1}{10} \sin \left(\frac{x \pi}{2}\right)+\frac{1}{3} \sin (x \pi+1)$, $\mu=0, \gamma=0.005, \Delta=1.3668 \ldots \ldots 26$

6 function $h(x)=\frac{\mu}{\gamma}(F+c)+\frac{5}{4} f, f(x)=\frac{1}{10} \sin \left(\frac{x \pi}{2}\right)+\frac{1}{3} \sin (x \pi+1)$, $F(x)=\frac{-1}{\pi}\left(\frac{1}{5} \cos \left(\frac{x \pi}{2}\right)+\frac{1}{3} \cos (x \pi+1)\right)$, with roots $\left(\alpha_{1}, \alpha_{2}, \alpha_{3}, \alpha_{4}\right)=$ $(0.7929,1.6190,2.6251,3.7222), \mu=0.0005, \gamma=0.005 \ldots 27$

7 comparison of the approximate solution for $\mu=0$ and $\mu=$ 0.0005 with $f(x)=\frac{1}{10} \sin \left(\frac{x \pi}{2}\right)+\frac{1}{3} \sin (x \pi+1), \gamma=0.005, \Delta=$

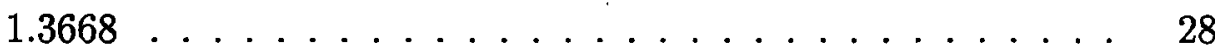

8 comparison of the numerical solution and leading order damped approximate solution for $f(x)=\frac{1}{10} \sin \left(\frac{x \pi}{2}\right)+\frac{1}{3} \sin (x \pi+1)$, $\mu=0.0005, \gamma=0.005, \Delta=1.3668 \ldots \ldots \ldots$

vii 
9 sample bifurcation diagram for $\mu=0.0005$ and $\gamma=0.005 \ldots$

10 peak pattern diagram for $f(x)=\frac{-3}{10} \cos \left(\frac{x \pi}{2}\right), \mu=0.0005, \gamma=$

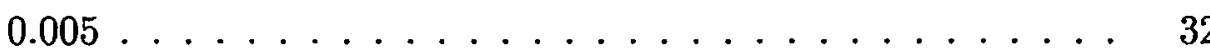

11 non-dispersive solution corresponding to $f(x)=\frac{1}{10} \sin \left(\frac{x \pi}{2}\right)+$ $\frac{1}{3} \sin (x \pi+1) \ldots \ldots \ldots \ldots \ldots \ldots \ldots$

12 peak pattern diagram for $f(x)=\frac{1}{10} \sin \left(\frac{x \pi}{2}\right)^{2}+\frac{1}{3} \sin (x \pi+1)$, $\mu=0.0005, \gamma=0.005 \ldots \ldots \ldots \ldots \ldots \ldots$

13 non-dispersive solution corresponding to the forcing in the second case $\ldots \ldots \ldots \ldots \ldots \ldots \ldots \ldots$

14 peak pattern diagram for the second case with $\mu=0.0005$ and $\gamma=0.005 \ldots \ldots \ldots \ldots \ldots \ldots \ldots$

15 potential barrier of the non-dispersive solution for the second case 52

16 potential barrier of the non-dispersive solution for the first case

17 non-dispersive solution with two equal global maxima corresponding to the first special case . . . . . . . . . .

18 peak pattern diagram for the first special case with $\mu=0.0005$ and $\gamma=0.005 \ldots \ldots \ldots \ldots \ldots \ldots \ldots \ldots$

19 potential barrier of the non-dispersive solution for the first special case . . . . . . . . . . . . . . .

viii 
20 non-dispersive solution corresponding to $f(x)=\frac{1}{10} \sin \left(\frac{x \pi}{2}\right)+$ $\frac{1}{3} \sin (x \pi) \ldots \ldots \ldots \ldots \ldots \ldots \ldots$

21 peak pattern diagram for $f(x)=\frac{1}{10} \sin \left(\frac{x \pi}{2}\right)+\frac{1}{3} \sin (x \pi-0.01)$, $\mu=0.0005, \gamma=0.005 \ldots \ldots \ldots \ldots \ldots$

22 sample $h(x)$ for $c=c_{\min }$ and $c=c_{\max }$, with $\mu=0.0005, \gamma=0.00557$

23 bifurcation diagram indicating regions of stability for $f(x)=$ $\frac{-3}{10} \cos \left(\frac{x \pi}{2}\right), \mu=0.0005, \gamma=0.005 \ldots \ldots \ldots 57$

24 peak pattern diagram with unstable solutions indicated by '*' symbols and stable solutions by points, for $f(x)=\frac{-3}{10} \cos \left(\frac{x \pi}{2}\right)$, $\mu=0.0005, \gamma=0.005 \ldots \ldots \ldots \ldots \ldots$

25 bifurcation diagram indicating regions of stability for $f(x)=$ $\frac{1}{10} \sin \left(\frac{x \pi}{2}\right)+\frac{1}{3} \sin (x \pi+1), \mu=0.0005, \gamma=0.005 \ldots 59$

26 peak pattern diagram with unstable solutions indicated by '*' symbols and stable solutions by points, for $f(x)=\frac{1}{10} \sin \left(\frac{x \pi}{2}\right)+$ $\frac{1}{3} \sin (x \pi+1), \mu=0.0005, \gamma=0.005 \ldots \ldots 60$ 


\section{Introduction}

The study of the general KdV equation [14] began in 1895 with the work of Korteweg and de Vries who derived a nonlinear equation governing waves in shallow water. Since then there have been many significant developments, including work by Miura, Gardner, and Kruskal [17] who found the existence of exact, localized travelling wave solutions, called solitons. This in turn led to the more general inverse scattering techniques which have been applied to many other nonlinear wave problems.

Much less is known about the forced KdV equation (fKdV), and thus yields a broad area of study. Physical examples of the fKdV equation can be seen in Grimshaw and Yi [12] where atmospheric flows are examined. Other examples include steady two layer flows over an obstacle presented by Dias and VandenBroeck [9], and free surface flow under gravity and surface tension discussed by Maleewong, Asavanant, and Grimshaw [15]. This paper is concerned with steady state solutions of the periodically forced $\mathrm{KdV}$ equation.

The study of steady state solutions of the periodically forced $\mathrm{KdV}$ equation began with the physical investigation of Chester and Bones [6], who considered the resonant forced oscillations of shallow water in a tank closed at both ends. Chester [5] proposed a theory to describe the steady state resonant forcing oscillations of a liquid in a closed rectangular tank. It was shown that near a resonant frequency, where linearized theory is invalid, the boundary conditions cannot be satisfied without the inclusion of the nonlinear term, although the oscillations can be described by the classical wave equation. The experimental 
results matched the numerical results of Chester [5]. The results showed that waves generated near the resonant frequency had high peaks separated by low troughs. The amplitude of the generated peaks was much larger than that of the wavemaker.

Ockendon and Ockendon [18] further investigated the physical problem using numerical methods which yielded much greater detail of the behaviour of the forced oscillating waves near the resonant frequency. Their study is the basis for the current mathematical approach to this problem.

By using multiple scale analysis, Cox and Mortell [7], [8] showed that the parametric continuation of the wave amplitude can be described by the periodically forced Korteweg-de Vries equation for resonant forcing about the fundamental, and by Amundsen et al. [3] for a periodic forcing about the half fundamental. Cox and Mortell [8] also presented numerical results for the parametric continuation of the resonant waves which confirmed the steady state solutions shown by the physical experiments.

Amundsen, Mortell, and Cox [4] demonstrated a method of constructing asymptotic solutions for small dispersion and damping. Through variation of the detuning parameter, families of resonant solutions were generated, providing precise depiction of the resonant solutions of the system, and allowing for direct comparison with the experimental results of Chester and Bones [6].

To date, investigation of the $\mathrm{fKdV}$ equation with periodic resonant forcing has been with the forcing being a single sinusoidal mode. This convenient forcing has been an integral part of the study of the fKdV equation, but in 
reality it is not fully representative of those forcings which might appear in a physical environment. It is more likely that forcings seen naturally would not be so simple. This thesis, in an effort to examine the problem for a general periodic forcing, addresses the case where the periodic resonant forcing becomes a function with several modes. This was accomplished by re-examining research of the problem while maintaining the forcing as a general forcing, $f(x)$. In terms of implementation it is convenient to represent the forcing $f(x)$ as a Fourier series.

In Section 2 the general matched, uniformly valid, leading order solutions for the general forcing, $f(x)$, are determined. These results are then used to determine the behaviour of the approximations through variation of the detuning parameter. They are examined in three subsections. In the first, the undamped case introduces the methods used to determine the leading order approximations. The second uses the same methods followed for the undamped case to determine the general matched, uniformly valid leading order solution for the damped case. Finally, the behaviour of the approximations through the parametric continuation of the detuning parameter is examined by describing peak formation and then peak movement within the resonant band of the detuning.

In Section 3 the numerical solutions are further investigated. First, approximations derived in Section 2 with given parameters and boundary conditions are calculated. These approximations are then compared to the corresponding numerical solution. Next, the numerical results over the entire spectrum of the detuning parameter are further explored. The discussion closely examines 
various classes of the general forcing, $f(x)$. The single mode forcing results are recreated to re-inforce the analytic discussion from Section 2.3. Following the single mode forcing, more general forcings are examined.

In Section 4 the stability of the numerical solutions and leading order approximations is discussed. First, the qualitative nature of the results which yield either stable or unstable solutions is described. Next, the bifurcation diagram examined in Section 2.3 is recreated with stability added to indicate the regions which yield stable and unstable solutions. Bifurcation diagrams from the classes of more general forcings from Section 3 are examined. The diagrams which track the qualitative behaviour of these specified forcings are also recreated, with the regions of stability indicated to support the results of the associated bifurcation diagrams.

To begin, the forced KdV equation must be simplified so that the general matched, uniformly valid leading order solutions can be determined. 


\section{Analytic Approximations}

In this section general matched, uniformly valid, leading order solutions are determined in a manner similar to [4] for the damped and undamped case. Then continuous variation of the solutions through the variation of the detuning parameter, $\Delta$, is examined. Consider the non-dimensionalized form of the forced KdV equation with Burgers' damping (fKdVB)

$$
U_{t}-\gamma U_{x x x}+\Delta U_{x}+\frac{3}{2} U U_{x}-\mu U_{x x}=f(x),
$$

over the domain $x \in[0,2 L]$ with periodic boundary conditions, a periodic function, $f(x)$, and subject to the zero mean condition

$$
\int_{0}^{2 L} U d x=0
$$

To find a steady state solution of $(2.1)$, taking $U_{t}=0$ yields

$$
-\gamma U_{x x x}+\Delta U_{\dot{x}}+\frac{3}{2} U U_{x}-\mu U_{x x}=f(x) .
$$

Further simplification can be made by taking $U=u-\frac{2}{3} \Delta$ so that the $\Delta U_{x}$ term is removed. This yields

$$
-\gamma u_{x x x}+\frac{3}{2} u u_{x}-\mu u_{x x}=f(x) .
$$

The mean value condition (2.2) in terms of $u$ becomes

$$
\frac{4 L}{3} \Delta=\int_{0}^{2 L} u d x
$$

The expression (2.3) may then be integrated with respect to $x$, and becomes

$$
\gamma u_{x x}=\frac{3}{4} u^{2}-\mu u_{x}-(F(x)+c),
$$

where $F(x)$ is an antiderivative of $f(x)$.

The cases where damping is present or absent can now be considered. 


\subsection{Undamped Case}

To determine the approximate solution for the undamped case, let $\mu=0$.

$$
\gamma u_{x x}=\frac{3}{4} u^{2}-(F(x)+c)
$$

A perturbation expansion $u=u_{0}+\sqrt{\gamma} u_{1}+O(\gamma)$ is introduced. Consider the solution in the outer layer, referred to as the non-dispersive solution, and assume $\gamma$ is small. Using (2.6), the perturbation expansion is applied, and to leading order (2.6) becomes

$$
0=\frac{3}{4} u_{0}^{2}-(F(x)+c)
$$

Solving for $u_{0}$ yields

$$
u_{0}= \pm \frac{2}{\sqrt{3}} \sqrt{F(x)+c}
$$

Henceforth, these will be referred to as the non-dispersive solutions

$$
u_{ \pm}= \pm \frac{2}{\sqrt{3}} \sqrt{F(x)+c}
$$

The value of the non-dispersive solutions, $u_{ \pm}$, must remain real, thus $c$ must satisfy

$$
\begin{aligned}
& F(x)+c>0 \text { for all } x \in[0,2 L], \\
& \text { where } \\
& c_{\text {min }}=\max _{x \in[0,2 L]}(-F(x)) .
\end{aligned}
$$

This leading order regular perturbation approximation does not satisfy the mean value condition (2.4) for certain values of $\Delta$ near zero. To address this, a singular perturbation approach is employed [13] and an inner boundary layer is 
introduced centred around a yet to be determined point $x=\alpha$. First, consider the case where the solution within the layer will take the form of a single peak. To further investigate the behaviour of the solution within the dispersive layer, (2.6) is re-scaled around $\alpha$ using the scaled variable

$$
X=\frac{x-\alpha}{\sqrt{\gamma}} \text { and } V(X)=u(x)
$$

As a consequence, to leading order $F(x)+c$ becomes $F(\alpha)+c=\beta+c$, where $\beta=F(\alpha)$. Introducing a perturbation expansion $V=V_{0}+\sqrt{\gamma} V_{1}+O(\gamma)$, to leading order (2.6) becomes

$$
V_{0 X X}=\frac{3}{4} V_{0}^{2}-(c+\beta)
$$

where at $\alpha, V_{0} \rightarrow u_{+}$as $X \rightarrow \pm \infty$. Solving for $V_{0}$ yields one of three general solutions; bounded oscillatory (multiple peaks), bounded (single peak), and unbounded (hypergeometric). Since the solution within the layer is a single peak, the general solution of interest is the bounded or the unbounded solution. As will be discussed in Section 4, the distinction between the two relates to stability of the solution. However, at leading order the analytic form of the solution is the same for both:

$$
V_{0}(x, X)=d+a \operatorname{sech}^{2}(k X)
$$

where

$$
a(x)=-8 k^{2}, \quad d(x)=\frac{8}{3} k^{2}, \quad k(x)=\left(\frac{3(F(x)+c)}{16}\right)^{\frac{1}{4}} .
$$

This becomes

$$
V_{0}=\frac{2}{\sqrt{3}} \sqrt{c+\beta}\left(1-3 \operatorname{sech}^{2}\left(\left(\frac{3(c+\beta)}{16}\right)^{\frac{1}{4}} X\right)\right) .
$$


In the original coordinate frame, the leading order solution in the inner layer is

$$
u=\frac{2}{\sqrt{3}} \sqrt{c+\beta}\left(1-3 \operatorname{sech}^{2}\left(\left(\frac{3(c+\beta)}{16}\right)^{\frac{1}{4}} \frac{x-\alpha}{\sqrt{\gamma}}\right)\right) .
$$

As a consequence of matching, (2.11) consists of two parts. The first is the leading order solution within the inner layer, that is

$$
u=-2 \sqrt{3} \sqrt{c+\beta}\left(\operatorname{sech}^{2}\left(\left(\frac{3(c+\beta)}{16}\right)^{\frac{1}{4}} \frac{x-\alpha}{\sqrt{\gamma}}\right)\right),
$$

and the second is the image of the non-dispersive solution (2.7)

$$
u=\frac{2}{\sqrt{3}} \sqrt{c+\beta}
$$

The solutions in the outer layer (2.7) and the inner layer (2.11) are matched, and yield a uniformly valid, leading order solution for the single peak case

$$
u=\frac{2}{\sqrt{3}} \sqrt{F(x)+c}-2 \sqrt{3} \sqrt{c+\beta} \operatorname{sech}^{2}\left(\left(\frac{3(c+\beta)}{16}\right)^{\frac{1}{4}} \frac{x-\alpha}{\sqrt{\gamma}}\right) .
$$

Using the mean value condition (2.4), $\Delta$ as a function of $u$ can be expressed as

$$
\begin{aligned}
\frac{4 L}{3} \Delta & =\int_{0}^{2 L} u d x \\
\Delta & =\frac{3}{4 L} \int_{0}^{2 L} u d x, \\
\Delta & =\frac{3}{4 L} \int_{0}^{2 L} u_{+} d x-\frac{3^{\frac{3}{4}}(c+\beta)^{\frac{1}{4}} \sqrt{\gamma}}{L} \tanh \left(\left(\frac{3(c+\beta)}{16}\right)^{\frac{1}{4}} \frac{2 L}{\sqrt{\gamma}}\right) .
\end{aligned}
$$

The value of $c$ in (2.12) is determined implicitly by (2.13) with a specified value of $\Delta$ in order to satisfy the mean value condition (2.4). In general, for a given value of $\Delta$, the existence of $c$ is not guaranteed. This will be seen in the bifurcation diagrams which will be discussed in Section 3.2. 
In general, it is also possible to have multiple peaks, but this requires a more detailed mathematical treatment. Although the boundary layer is small, it is not small enough. Variation of the forcing over the region is now significant. As a result, the following multiple scale analysis is applied.

$$
u(x) \rightarrow V(x, X)
$$

where

$$
X=\frac{\omega_{1}(x)}{\sqrt{\gamma}}+\omega_{2}(x)+\sqrt{\gamma} \omega_{3}(x)+O(\gamma)
$$

The function $\omega_{1}(x)$ will be solved later in this section. Subsequent $\omega$-functions are only necessary at higher order approximations, and are not needed for this analysis.

The differential operator for this multiple scale analysis becomes

$$
\frac{d}{d x} \rightarrow \frac{\partial}{\partial x}+\frac{\partial X}{\partial x} \frac{\partial}{\partial X}
$$

and substituting in the perturbation expansion (2.14), it is

$$
\frac{d}{d x}=\frac{\partial}{\partial x}+\left(\frac{\omega_{1}^{\prime}(x)}{\sqrt{\gamma}}+\omega_{2}^{\prime}(x)+\ldots\right) \frac{\partial}{\partial X} .
$$

Similarly, the second order operator is

$$
\begin{aligned}
\frac{d^{2}}{d x^{2}}= & \left(\frac{\partial}{\partial x}+\left(\frac{\omega_{1}^{\prime}(x)}{\sqrt{\gamma}}+\omega_{2}^{\prime}(x)+\ldots\right) \frac{\partial}{\partial X}\right)\left(\frac{\partial}{\partial x}+\left(\frac{\omega_{1}^{\prime}(x)}{\sqrt{\gamma}}+\omega_{2}^{\prime}(x)+\ldots\right) \frac{\partial}{\partial X}\right) \\
= & \frac{\partial^{2}}{\partial x^{2}}+2\left(\frac{\omega_{1}^{\prime}(x)}{\sqrt{\gamma}}+\omega_{2}^{\prime}(x)+\ldots\right) \frac{\partial^{2}}{\partial X \partial x} \\
& +\left(\frac{\omega_{1}^{\prime}(x)}{\sqrt{\gamma}}+\omega_{2}^{\prime}(x)+\ldots\right)^{2} \frac{\partial^{2}}{\partial X^{2}}+\left(\frac{\omega_{1}^{\prime \prime}(x)}{\sqrt{\gamma}}+\omega_{2}^{\prime \prime}(x)+\ldots\right) \frac{\partial}{\partial X} \\
= & \frac{1}{\gamma}\left(\omega_{1}^{\prime 2} \frac{\partial^{2}}{\partial X^{2}}\right)+\frac{1}{\sqrt{\gamma}}\left(2 \omega_{1}^{\prime} \frac{\partial^{2}}{\partial X \partial x}+2 \omega_{1}^{\prime} \omega_{2}^{\prime} \frac{\partial^{2}}{\partial X^{2}}+\omega_{1}^{\prime \prime} \frac{\partial}{\partial X}\right)+O(1)
\end{aligned}
$$


Now, when the multiple scale perturbation (2.14) and the stretched coordinates (2.9) used in the single peak case are applied to (2.6), the equation becomes

$$
\begin{aligned}
0= & \left(\omega_{1}^{\prime 2} V_{0 X X}-\frac{3}{4} V_{0}^{2}+F(x)+c\right) \\
& +\sqrt{\gamma}\left(\omega_{1}^{\prime 2} V_{1 X X}+2 \omega_{1}^{\prime} V_{0 x X}+2 \omega_{1}^{\prime} \omega_{2}^{\prime} V_{0 X X}+\omega_{1}^{\prime \prime} V_{0 X}-\frac{3}{2} V_{0} V_{1}\right) \\
& +O(\gamma) .
\end{aligned}
$$

Examining leading order $(O(1))$ yields

$$
\omega_{1}^{2} V_{0 X X}-\frac{3}{4} V_{0}^{2}+F(x)+c=0,
$$

where $V_{0} \rightarrow u_{+}$at the boundaries of the layer. The solution for this equation yields the same three general solutions as for the single peak case, only now the general solution within the layer is assumed to be oscillatory, yielding a solution in terms of a Jacobi elliptic function

$$
V_{0}(x, X)=d+a \operatorname{cn}(k(X), m(x))^{2}
$$

where $\mathrm{cn}$ is a cnoidal function, and

$$
a(x)=-8 \omega_{1}^{\prime 2} m^{2} k^{2}, d(x)=\frac{8}{3} \omega_{1}^{\prime 2} k^{2}\left(2 m^{2}-1\right), k(x)=\left(\frac{3(F(x)+c)}{16 \omega_{1}^{4}\left(m^{4}-m^{2}+1\right)}\right)^{\frac{1}{4}} .
$$

To determine the spatial variation of the modulus $m(x)$, the next order term $(O(\sqrt{\gamma}))$ is examined.

$$
\begin{aligned}
& \omega_{1}^{\prime 2} V_{1 X X}+2 \omega_{1}^{\prime} V_{0 x X}+2 \omega_{1}^{\prime} \omega_{2}^{\prime} V_{0 X X}+\omega_{1}^{\prime \prime} V_{0 X}-\frac{3}{2} V_{0} V_{1}=0 \\
& \omega_{1}^{\prime 2} V_{1 X X}-\frac{3}{2} V_{0} V_{1}=-2 \omega_{1}^{\prime} V_{0 x X}-2 \omega_{1}^{\prime} \omega_{2}^{\prime} V_{0 X X}-\omega_{1}^{\prime \prime} V_{0 X} .
\end{aligned}
$$

In order to avoid secularity $V_{0}$ must be independent of $x$ over its period, otherwise linear growth would arise in $X$. As a result, the period $T$ is an 
arbitrary constant and is expressed as

$$
T=\frac{2 K(m)}{k}=4 K(m) \omega_{1}^{\prime}\left(\frac{\left(m^{4}-m^{2}+1\right)}{3(F(x)+c)}\right)^{\frac{1}{4}} .
$$

Since $T$ is arbitrary, for convenience let $T=\frac{4}{3^{\frac{1}{4}}}$. Since $m=m(x), \omega_{1}^{\prime}$ is determined so that it will cancel with all $x$-dependent factors in (2.17). Therefore

$$
\omega_{1}^{\prime}=\frac{1}{K(m)}\left(\frac{F(x)+c}{m^{4}-m^{2}+1}\right)^{\frac{1}{4}}
$$

Multiplying (2.16) by $V_{0 X}$ (the solution to the adjoint equation) and integrating over a single period yields

$$
\left.V_{0 X} V_{1 X}\right|_{0} ^{T}-\left.V_{0 X X} V_{1}\right|_{0} ^{T}+\int_{0}^{T} \omega_{1}^{\prime 2} V_{0 X X X} V_{1}-\frac{3}{2} V_{0} V_{0 X} V_{1} d X=\int_{0}^{T} V_{0 X}(\mathrm{RHS}) d X
$$

By periodicity, the first two terms vanish, so

$$
\int_{0}^{T} V_{1}\left(\omega_{1}^{\prime 2} V_{0 X X X}-\frac{3}{2} V_{0} V_{0 X}\right) d X=\int_{0}^{T} V_{0 X}(\mathrm{RHS}) d X
$$

Next, differentiation of (2.15) implies that the integrand on the left side vanishes, so all that remains is

$$
\begin{aligned}
0 & =\int_{0}^{T} V_{0 X}(\mathrm{RHS}) d X \\
& =\int_{0}^{T}-2 \omega_{1}^{\prime} V_{0 x X} V_{0 X}-2 \omega_{1}^{\prime} \omega_{2}^{\prime} V_{0 X X} V_{0 X}-\omega_{1}^{\prime \prime} V_{0 X}^{2} d X
\end{aligned}
$$

First consider the integral

$$
\begin{aligned}
\int_{0}^{T}-2 \omega_{1}^{\prime} \omega_{2}^{\prime} V_{0 X X} V_{0 X} d X & =-2 \omega_{1}^{\prime} \omega_{2}^{\prime} \int_{0}^{T} V_{0 X X} V_{0 X} d X \\
& =-2 \omega_{1}^{\prime} \omega_{2}^{\prime} \int_{0}^{T} \frac{d}{d X}\left(\frac{V_{0 X}^{2}}{2}\right) d X \\
& =-\omega_{1}^{\prime} \omega_{2}^{\prime}\left(V_{0 X}^{2}(T)-V_{0 X}^{2}(0)\right) \\
& =0 \text { (by periodicity) }
\end{aligned}
$$


All that remains in $(2.19)$ is

$$
\begin{aligned}
0 & =\int_{0}^{T}-2 \omega_{1}^{\prime} V_{0 x X} V_{0 X}-\omega_{1}^{\prime \prime} V_{0 X}^{2} d X \\
& =-\int_{0}^{T}\left(\omega_{1}^{\prime} V_{0 X}^{2}\right)_{x} d X \\
& =-\frac{d}{d x} \int_{0}^{T} \omega_{1}^{\prime} V_{0 X}^{2} d X
\end{aligned}
$$

which can be expressed as

$$
\frac{d Q}{d x}=0
$$

where

$$
Q=\int_{0}^{T} \omega_{1}^{\prime} V_{0 X}^{2} d X
$$

and thus

$$
\omega_{1}^{\prime} \int_{0}^{T} V_{0 X}^{2} d X=\kappa
$$

where $\kappa$ is a constant. Using the known expression for $\omega_{1}^{\prime},(2.18)$, and the properties of elliptic functions, $m$ can be implicitly determined as the solution of

$$
\frac{16(3)^{\frac{1}{4}}}{5} \frac{(F(x)+c)^{\frac{5}{4}}}{\left(m^{4}-m^{2}+1\right)^{\frac{1}{4}}}\left(2 E(m)-\frac{\left(1-m^{2}\right)\left(2-m^{2}\right)}{m^{4}-m^{2}+1} K(m)\right)=\kappa .
$$

To further understand the behaviour of the solution within the dispersive layer, (2.23) is re-arranged into the form $G(m)=g(x)$

$$
\frac{\kappa 16(3)^{\frac{1}{4}}}{5\left(m^{4}-m^{2}+1\right)^{\frac{1}{4}}}\left(2 E(m)-\frac{\left(1-m^{2}\right)\left(2-m^{2}\right)}{m^{4}-m^{2}+1} K(m)\right)=\frac{1}{(F(x)+c)^{\frac{5}{4}}} .
$$

By the relationship $G(m)=g(x)$, and to satisfy matching criteria on either side of the dispersive layer, the function within the layer must contain a maximum or a minimum of the non-dispersive solution, (2.7). Therefore, the inner 
layer is located at a maximum or a minimum of the non-dispersive solution (Amundsen, Cox, and Mortell [4]).

In general, dispersive layers may exist at any of the local maxima and/or minima of the non-dispersive solution, and their existence is subject to the mean value condition (2.4). Thus the set of roots $\alpha_{i}$, and the corresponding $\beta_{i}=F\left(\alpha_{i}\right)$, for $i=1,2, \ldots, n$ yield the general matched, uniformly valid, leading order solution

$$
u=\frac{2}{\sqrt{3}} \sqrt{F(x)+c}-2 \sqrt{3} \sum_{i=1}^{n} \sqrt{c+\beta_{i}} \Phi_{i}(x)
$$

where

$$
\Phi_{i}(x)=\left\{\begin{array}{l}
\operatorname{sech}^{2}\left(\left(\frac{3\left(c+\beta_{i}\right)}{16}\right)^{\frac{1}{4}} \frac{x-\alpha_{i}}{\sqrt{\gamma}}\right) \text { for the single peak case } \\
\operatorname{cn}\left(k(x)\left(\frac{\omega_{1}(x)}{\sqrt{\gamma}}\right), m(x)\right)^{2} \text { for the multiple peak case }
\end{array}\right.
$$

subject to $\Phi_{i}(x) \rightarrow 0$ outside the dispersive layer.

For the single peak case, the value of $c$ is determined implicitly by (2.13). In the multiple peak case the expression

$$
\Delta=\frac{3}{4 L} \int_{0}^{2 L} u d x
$$

cannot be expressed analytically, so in practice $c$ needs to be determined numerically.

Now that the leading order approximation for the undamped case has been determined, the leading order approximation for the damped case is considered. 


\subsection{Damped Case}

To determine the approximate solution for the damped case, consider the equation (2.5). For this thesis the damping coefficient $\mu$ is considered to be of the same order as the dispersive parameter $\gamma$. Though it is not always the case in all physical contexts, the assumption that $\mu=O(\gamma)$ is also made in previous work, as stated by example in Amundsen, Cox, and Mortell [4], Malkov [16], and Ockendon and Ockendon [18], and has been shown to correspond to the experiments of Chester and Bones [6]. The combination of the dispersive and the non-dispersive solutions, where $c$ is determined for a given $\Delta$, satisfies the mean value condition (2.4). To determine the non-dispersive solution, the perturbation $u=u_{0}+\sqrt{\gamma} u_{1}+O(\gamma)$ applied to leading order (2.5) yields

$$
0=\frac{3}{4} u_{0}^{2}-(F(x)+c)
$$

Solving for $u_{0}$ leads to the same non-dispersive solution as for the undamped case

$$
u_{ \pm}= \pm \frac{2}{\sqrt{3}} \sqrt{(F(x)+c)}
$$

To determine the dispersive solution, the same perturbation (2.14) and stretching (2.9) are applied to (2.5). The same solution is reached at leading order, but at $O(\sqrt{\gamma})$ the expansion yields

$$
\omega_{1}^{\prime 2} V_{1 X X}-\frac{3}{2} V_{0} V_{1}=-2 \omega_{1}^{\prime} V_{0 x X}-2 \omega_{1}^{\prime} \omega_{2}^{\prime} V_{0 X X}-\omega_{1}^{\prime \prime} V_{0 X}-\frac{\mu}{\gamma} \omega_{1}^{\prime} V_{0 X}
$$

Following the same arguments as for the undamped case (2.19) only now with damping being included, the following solvability condition must be satisfied

$$
0=\int_{0}^{T}-2 \omega_{1}^{\prime} V_{0 x X} V_{0 X}-2 \omega_{1}^{\prime} \omega_{2}^{\prime} V_{0 X X} V_{0 X}-\omega_{1}^{\prime \prime} V_{0 X}^{2}-\frac{\mu}{\gamma} \omega_{1}^{\prime} V_{0 X}^{2} d X
$$


Using (2.20) and (2.21) it simplifies to

$$
0=\int_{0}^{T}-2 \omega_{1}^{\prime} V_{0 x X} V_{0 X}-\omega_{1}^{\prime \prime} V_{0 X}^{2} d X-\frac{\mu}{\gamma} \int_{0}^{T} \omega_{1}^{\prime} V_{0 X}^{2} d X
$$

which is equivalent to

$$
\frac{d Q}{d x}+\frac{\mu}{\gamma} Q=0
$$

where

$$
Q=\int_{0}^{T} \omega_{1}^{\prime} V_{0 X}^{2} d X
$$

The solution of $(2.26)$ is

$$
Q=\kappa e^{-\frac{\mu}{\gamma} x}
$$

which is consistent with (2.22) if $\mu=0$. This expression is solved in the same manner as (2.23) but yields

$$
\frac{16(3)^{\frac{1}{4}}}{5} \frac{(F(x)+c)^{\frac{5}{4}}}{\left(m^{4}-m^{2}+1\right)^{\frac{1}{4}}}\left(2 E(m)-\frac{\left(1-m^{2}\right)\left(2-m^{2}\right)}{m^{4}-m^{2}+1} K(m)\right)=\kappa e^{-\frac{\mu}{\gamma} x} .
$$

From this equation, $m$ can be determined implicitly for the damped case. In order to match, the expression (2.27) is re-arranged to the form $G(m)=g(x)$

$$
\frac{\kappa 16(3)^{\frac{1}{4}}}{5\left(m^{4}-m^{2}+1\right)^{\frac{1}{4}}}\left(2 E(m)-\frac{\left(1-m^{2}\right)\left(2-m^{2}\right)}{m^{4}-m^{2}+1} K(m)\right)=\frac{e^{-\frac{\mu}{\gamma} x}}{(F(x)+c)^{\frac{5}{4}}} .
$$

So now

$$
G(m)=g(x)=\frac{e^{-\frac{\mu}{\gamma} x}}{(F+c)^{\frac{5}{4}}} .
$$

By satisfying the same matching criteria as in the undamped case, $G(m)=$ $g(x)$, the inner and outer layers are matched about a maximum or minimum of $g(x)$. In the undamped case, determining the roots of $g^{\prime}(x)$ is equivalent to determining the roots of the non-dispersive solution (2.7), and this yielded the peak locations, $\alpha_{i}$. In the damped case, the solution to the differential equation 
(2.26) is no longer a constant. The damped case differs from the undamped case in that the value of the non-dispersive solution where matching occurs is no longer equal in the two surrounding boundary layers. The matching criteria in the boundary layers surrounding the inner layer now differ by a factor of $e^{-\frac{\mu}{\gamma} x}$. The peaks will be shifted from maxima/minima of the non-dispersive solution, but the roots of $g^{\prime}(x)$ will still yield their location.

$$
0=g^{\prime}(x)=-\frac{\mu}{\gamma} e^{-\frac{\mu}{\gamma} x}(F+c)^{-\frac{5}{4}}-\frac{5}{4} e^{-\frac{\mu}{\gamma} x}(F+c)^{-\frac{9}{4}} f .
$$

Since $F(x)+c \neq 0$, this simplifies to the following expression

$$
0=h(x)=\frac{\mu}{\gamma}(F+c)+\frac{5}{4} f .
$$

The roots of $h(x)$ correspond to the points where the peaks are centred for both the damped and undamped case. The roots, $x=\alpha_{i}$, have corresponding values $\beta_{i}=F\left(\alpha_{i}\right)$ for $i=1,2, \ldots, n$. This yields the same general matched, uniformly valid leading order solution

$$
u=\frac{2}{\sqrt{3}} \sqrt{F(x)+c}-2 \sqrt{3} \sum_{i=1}^{n} \sqrt{c+\beta_{i}} \Phi_{i}(x)
$$

where

$$
\Phi_{i}(x)=\left\{\begin{array}{l}
\operatorname{sech}^{2}\left(\left(\frac{3\left(c+\beta_{i}\right)}{16}\right)^{\frac{1}{4}} \frac{x-\alpha_{i}}{\sqrt{\gamma}}\right) \text { for the single peak case } \\
\operatorname{cn}\left(k(x)\left(\frac{\omega_{1}(x)}{\sqrt{\gamma}}\right), m(x)\right)^{2} \text { for the multiple peak case, }
\end{array}\right.
$$

subject to $\Phi_{i}(x) \rightarrow 0$ outside the dispersive layer.

The values for $c$ are determined in the same way as in the undamped case, using

$$
\Delta=\frac{3}{4 L} \int_{0}^{2 L} u d x
$$


This solution yields the same result as Section 2.1, except that the peaks for the damped case are roots of $h(x)$, not $f(x)$. However, as stated, the roots of $h(x)$ will yield the peak location for the undamped case because if $\mu=0, h(x)$ and $f(x)$ have the same roots. For this reason, the solutions for this section can be effectively treated as the general matched, uniformly valid, leading order solution to (2.1) for a given $\Delta$ in both damped and undamped cases.

Now that the leading order approximations can be determined for specific initial conditions, the continuous variation of the solutions by variation of the detuning parameter, $\Delta$, is examined.

\subsection{Peak Formation and Movement}

As discussed in Sections 2.1 and 2.2, the value of $c$ is dependent on $\Delta$ and $u$ by (2.13). Thus for any given $\Delta$, only a discrete set of solutions may exist. These solutions can be explained by continuous variation of solutions as $\Delta$ is varied. For sufficiently large $\Delta$, the mean value condition (2.4) is satisfied by the non-dispersive solution (2.7). The limiting case of $\Delta$ arises when $c=c_{\min }$, and is given by

$$
\Delta^{*}=\lim _{c \rightarrow c_{\min }} \Delta,
$$

for $u=u_{+}$and $u=u_{-}$. This yields the bound of the resonant band. For $\Delta>\Delta^{*}$ the solution can be $u=u_{+}$, and for $\Delta<-\Delta^{*}$ the solution can be $u=u_{-}$. For $|\Delta|<\Delta^{*}$ the non-dispersive solution is not sufficient to satisfy the mean value condition, so for this reason dispersive layers are generated within the resonant band. 


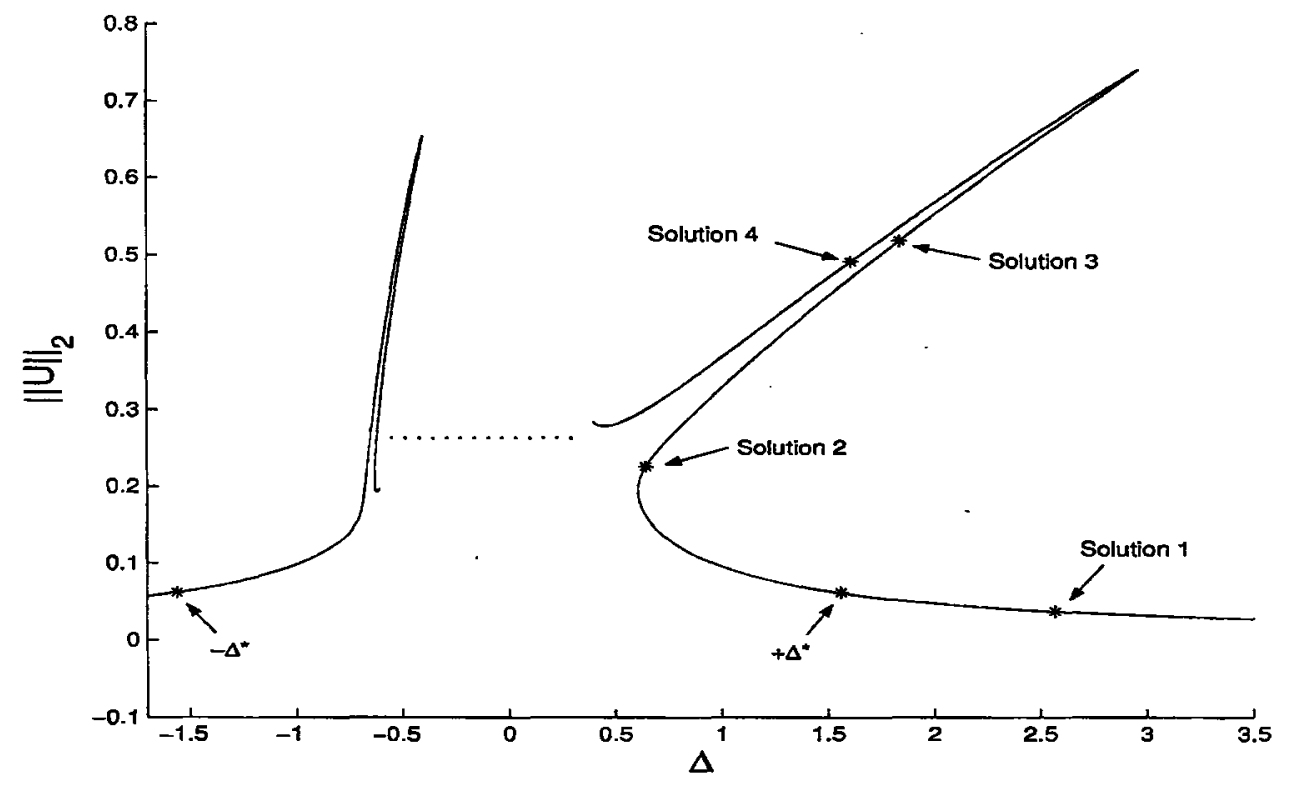

Figure 1: partial finger diagram illustrating known behaviour in the resonant band

Figure 1 is a partial bifurcation diagram which illustrates the nature of the bifurcation diagram of the solutions. One of the primary purposes of this thesis, examination of the complete bifurcation diagram, is discussed in Section 3.2. Parametric continuation of the solution within the resonant band yields the transition from $u_{-}$to $u_{+}$. As $\Delta$ varies, $c$ must be large enough to satisfy (2.8). Consider a single mode forcing which yields a non-dispersive solution such as Figure 2. To determine the peak location consider the limiting case when $c=c_{\text {min }}$. There exists a point $x_{i}$ such that a cusp will form at the global minimum of the non-dispersive solution satisfying

$$
F\left(x_{i}\right)+c_{\min }=0
$$

while $\sqrt{F(x)+c}$ is still real. For non-dispersive solutions with multiple maxima and minima, cusps will not form at any other minima since $c$ is constrained 


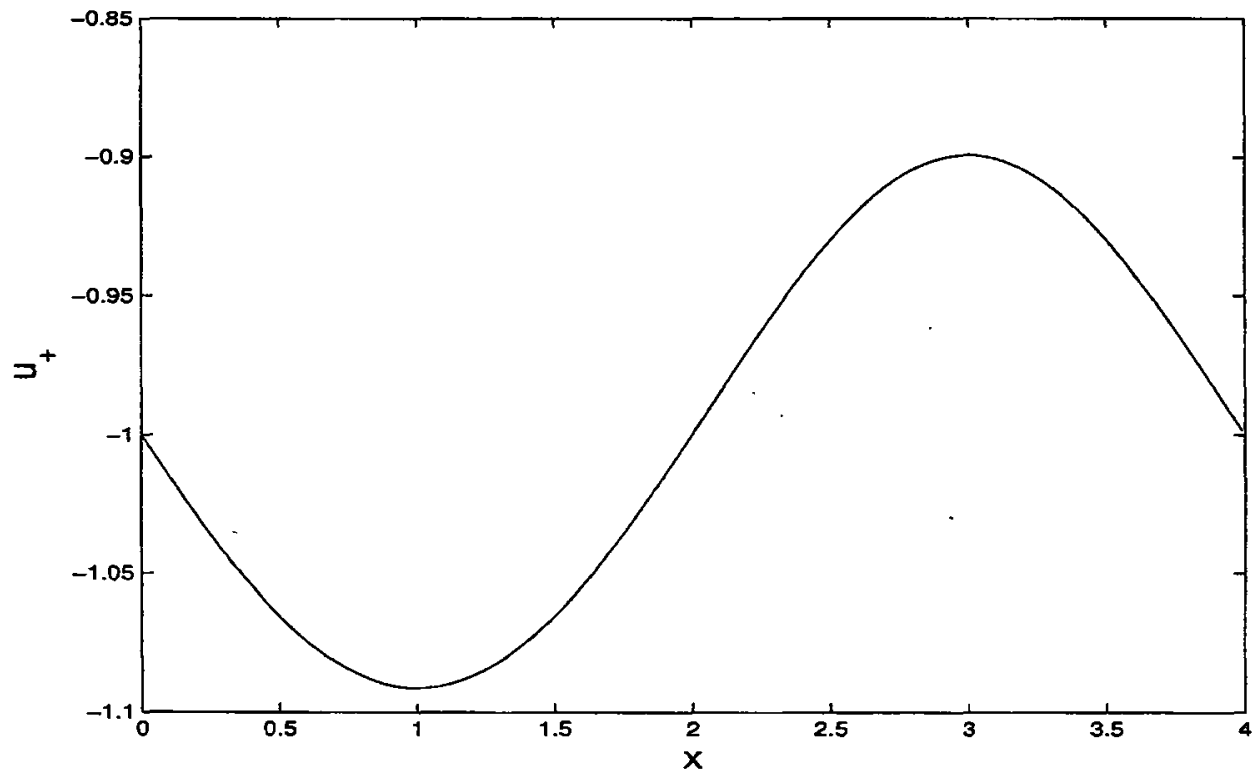

Figure 2: non-dispersive solution derived from sample single mode forcing by the global minimum of $F(x)$. The location of the cusp, $x_{i}$, is a root of $f(x)$ which corresponds to the global minimum of the non-dispersive solution. The peak is created at the cusp to ensure the solution remains smooth and differentiable. As the solutions evolve through varying $\Delta$, the peaks which are created must remain in order to continue satisfying the zero mean condition, (2.4).

Considering the damped case, the peaks are generated at $\alpha_{i}$, a root of $h(x)$ which is near $x_{i}$, such that the function remains smooth and differentiable. The slope at this root is positive because $h(x)$ is a function of $F(x)$, the antiderivative of $f(x)$. For $\mu$ small and positive, the given $\alpha_{i}$ corresponds to a point slightly to the left of the global minimum of the non-dispersive solution. As $\Delta$ increases $c$ also increases by (2.13), and the peak begins to move towards an adjacent maximum. To satisfy the mean value condition 
(2.4), the amplitude of the peak grows to compensate for the upward shift of the solution in the non-dispersive layer. While $\Delta$ is increasing, for the peak to move in a continuous manner it must move to the left. Equivalently, if $\mu$ were small and negative, the peak would be created slightly to the right of the global minimum of the non-dispersive solution, and while $\Delta$ is increasing, the peak would shift to the right.
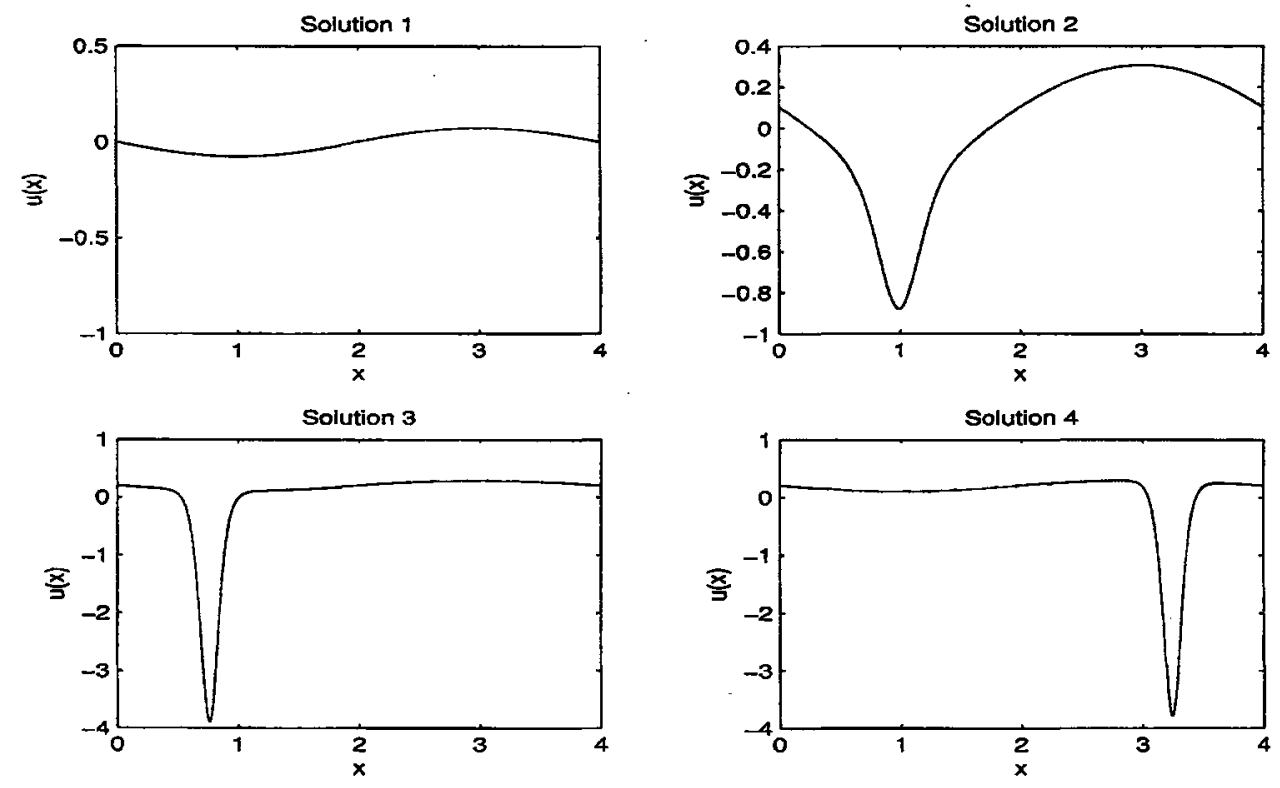

Figure 3: solutions corresponding to each of the four points on the partial bifurcation diagram, Figure 1

The progression of the first peak is illustrated on the partial bifurcation diagram, Figure 1, with solutions of each point shown on Figure 3. The first solution corresponds to a value of $\Delta$ outside the resonant band. The second corresponds to a $\Delta$ when the first peak is formed. The third solution shows how the peak has begun to move to the left, and its amplitude has increased. The fourth yields a solution where the peak is positioned near the adjacent maximum. Subsequent variation of $\Delta$ will in turn yield more peaks which 
form around the minimum and accumulate at the maximum.

Now that leading order approximations have been determined, and the creation and movement of the peaks through variation of the detuning parameter is generally understood, the numerical methods are used to verify the results and continue the understanding of the problem through the variation of the detuning parameter. 


\section{$3 \quad$ Numerical Examples}

In this section approximate solutions are compared to numerical solutions for a specified $\Delta$. Next, the parametric continuation of various forcings is examined. Section 3.1 shows particular damped and undamped leading order approximations which satisfy the mean value condition (2.4). Then, the damped approximation of the solution is compared to the numerical solution. Section 3.2 examines the steady solutions arising for more general forcings corresponding to non-dispersive solutions with various arrangements of the maxima and minima.

\subsection{Comparison of Analytic Approximations and $\mathrm{Nu}-$ merical Solutions}

As shown in Section 2.1 and 2.2, analytic approximations can be used to create a particular approximation from any forcing with given parameters. Peaks are created at the global minimum of the non-dispersive solution and then move to the left through variation of the detuning parameter. As will be further discussed in Section 4, the peaks are deposited near the maximum to the immediate left of the global minimum of the non-dispersive solution. The location of such a peak is $\alpha_{i}$, the corresponding root of $f(x)$ (or $h(x)$ for the damped case), and it follows that the slope at $f\left(\alpha_{i}\right)$ is negative. Then, with a given value of $\Delta$, the corresponding value of $c$ can be determined using (2.7). Finally, determining the value $\beta_{i}=F\left(\alpha_{i}\right)$, and with given initial conditions $\gamma$ and $\mu$, the approximate solution can be created using (2.29) and (2.30). 
For example, consider the forcing

$$
f(x)=\frac{1}{10} \sin \left(\frac{x \pi}{2}\right)+\frac{1}{3} \sin (x \pi+1)
$$

with $\gamma=0.005, \mu=0.0005$, and $\Delta=1.3668$. Corresponding to this forcing an antiderivative, $F(x)$, is

$$
F(x)=\frac{-1}{\pi}\left(\frac{1}{5} \cos \left(\frac{x \pi}{2}\right)+\frac{1}{3} \cos (x \pi+1)\right) .
$$

A well known computational package for parametric continuation of dynamical systems, AUTO 97 [10], is used to parametrically continue (2.1) for specified forcings and values for $\gamma$ and $\mu$. The program uses a spectral decomposition to solve the system of ordinary differential equations which corresponds to the fKdV equation (2.1), for specified parameter values and a given forcing, yielding

$$
u=\sum_{n=1}^{N} A_{n} \cos \frac{n \pi x}{L}+B_{n} \sin \frac{n \pi x}{L},
$$

where $A_{n}$ and $B_{n}$ are the Fourier coefficients. It was found that taking $\mathrm{N}=40$ was more than sufficient to produce accurate numerical solutions. The damped solutions were generated as $\Delta$ was varied, and are matched to the analytic approximations. Sample numerical code and all visualizations used in this thesis are provided on the affixed $\mathrm{CD}$. Choosing an example with a single peak, the solution selected corresponds to $\Delta=1.3668$ and uses the forcing given above. The roots of this forcing (equivalently, the maxima of the nondispersive solution (2.7)) must be determined.

$$
0=F^{\prime}(x)=f(x)=\frac{1}{10} \sin \left(\frac{x \pi}{2}\right)+\frac{1}{3} \sin (x \pi+1),
$$

where the roots are

$$
\left(\alpha_{1}, \alpha_{2}, \alpha_{3}, \alpha_{4}\right) \doteq(0.7724,1.6289,2.6033,3.7222)
$$




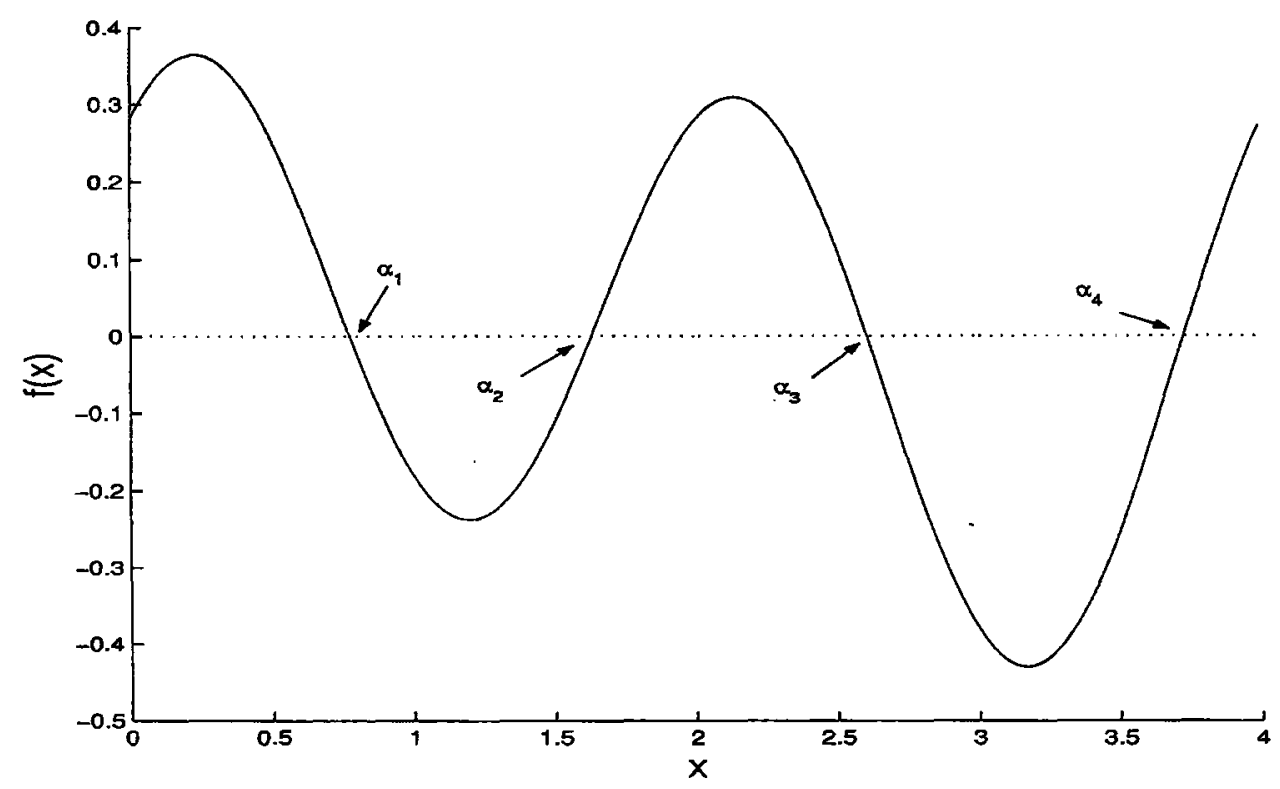

Figure 4: forcing $f(x)=\frac{1}{10} \sin \left(\frac{x \pi}{2}\right)+\frac{1}{3} \sin (x \pi+1)$, with roots $\left(\alpha_{1}, \alpha_{2}, \alpha_{3}, \alpha_{4}\right)=(0.7724,1.6288,2.6034,3.7221)$

As shown in Figure 4, the roots of $f(x)$ where the slope $f\left(\alpha_{i}\right)$ is positive corresponds to the minima in the non-dispersive solution. The root with the greatest slope corresponds to the global minimum, which in this case is $\alpha_{4}$. Conversely, the roots of $f(x)$ where the slope $f\left(\alpha_{i}\right)$ is negative corresponds to the maxima. These two roots are $\alpha_{1}$ and $\alpha_{3}$. The maximum to the immediate left of the global minimum corresponds to the root $\alpha_{3}$. Using (2.13), the value of $c=0.9089$ is determined for the given value of $\Delta$. Next,

$$
c-\beta=c-F\left(\alpha_{3}\right)=c-F(2.60)
$$

yields

$$
\beta=F\left(\alpha_{3}\right)=0.1400
$$

Substituting these values into (2.29) and (2.30) yields the resulting leading order undamped steady state approximation, Figure 5. 


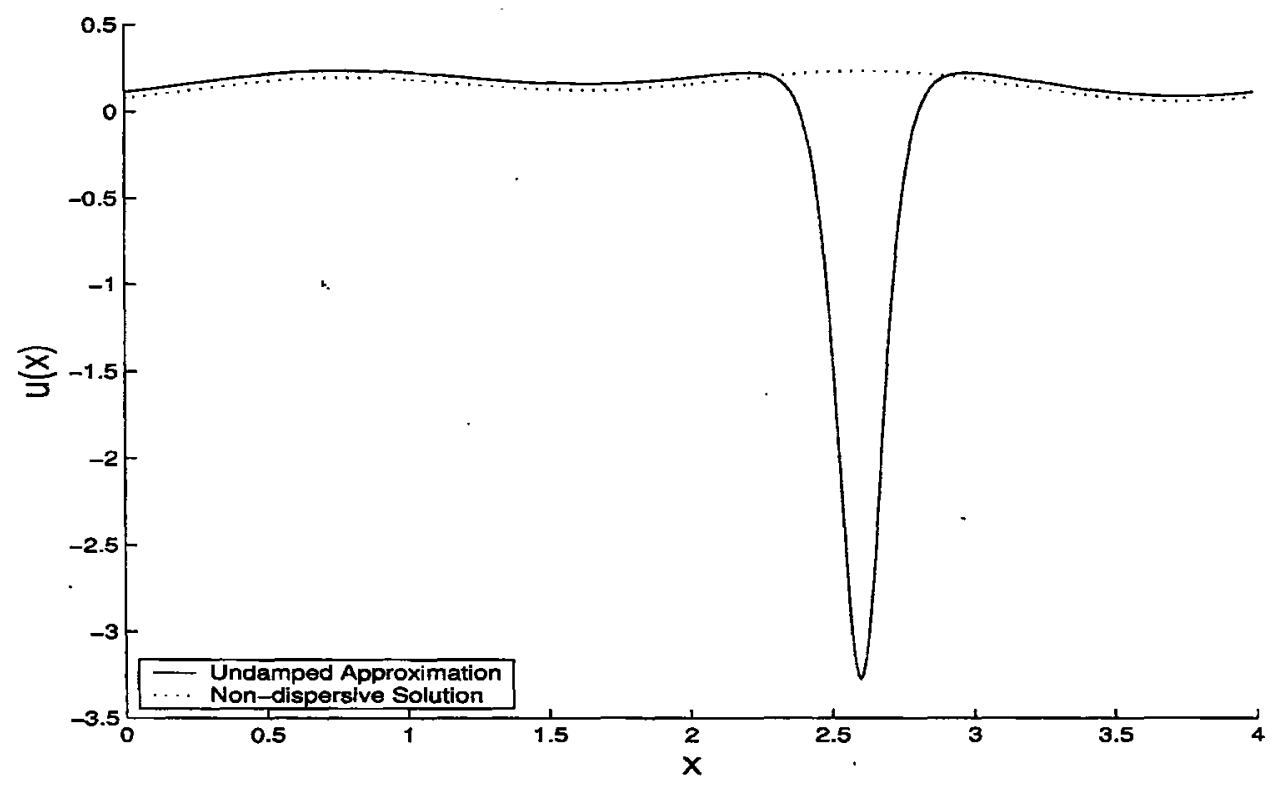

Figure 5: approximate solution for $f(x)=\frac{1}{10} \sin \left(\frac{x \pi}{2}\right)+\frac{1}{3} \sin (x \pi+1), \mu=0$, $\gamma=0.005, \Delta=1.3668$

The same steps are followed for the approximate damped solution, with the only difference being the peak location. The roots of $h(x)$ are

$$
\left(\alpha_{1}, \alpha_{2}, \alpha_{3}, \alpha_{4}\right) \doteq(0.7929,1.6190,2.6251,3.7222)
$$

as seen in Figure 6. The root to the immediate left of the global minimum of the non-dispersive solution is $\alpha_{3}=2.6251$, and has corresponding

$$
\beta=F\left(\alpha_{3}\right)=0.1376
$$

The new value of $\beta$ and same value $\Delta$ are used in (2.13) to yield $c=0.9807$. Using (2.29) and (2.30) the approximate leading order damped solution is plotted along with the original undamped approximate solution, shown in Figure 7. This figure shows how the peak location for the damped case differs from the undamped case, as discussed in Section 2.2. The damped analytic 


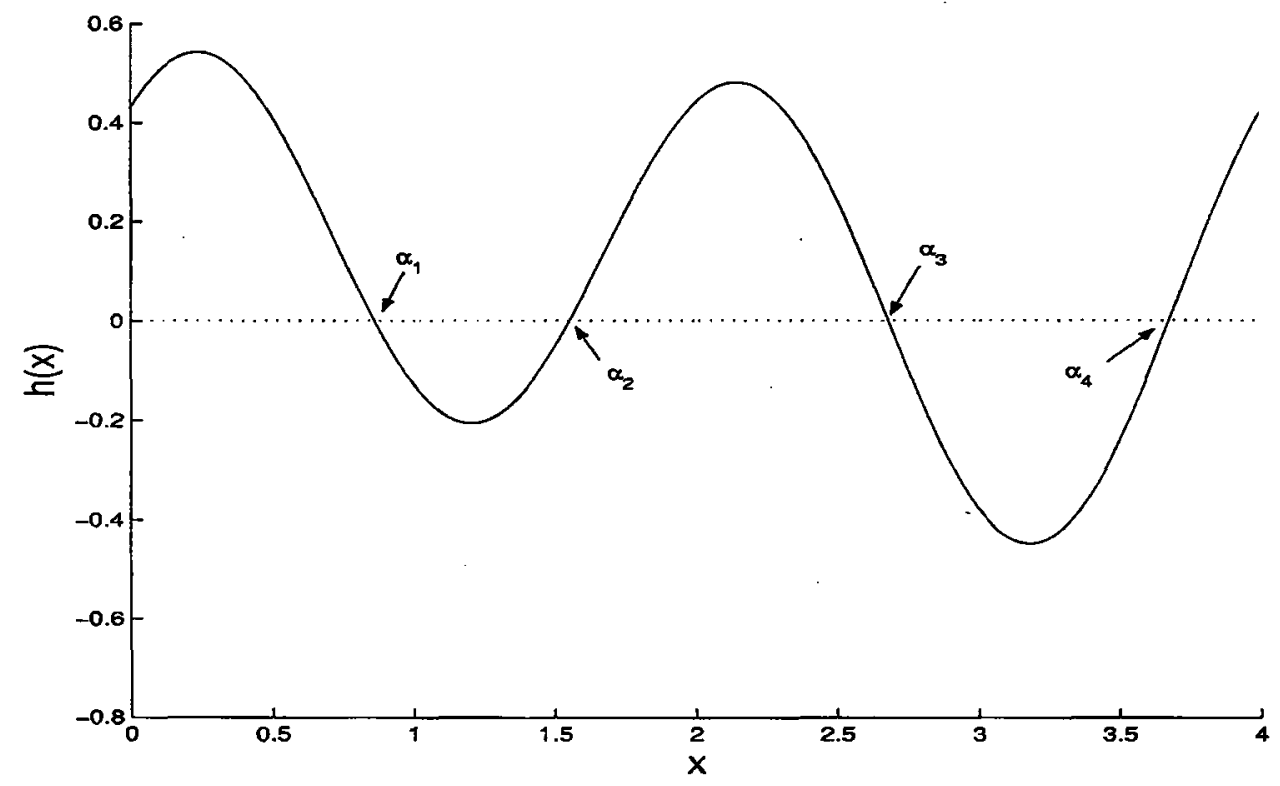

Figure 6: function $h(x)=\frac{\mu}{\gamma}(F+c)+\frac{5}{4} f, f(x)=\frac{1}{10} \sin \left(\frac{x \pi}{2}\right)+\frac{1}{3} \sin (x \pi+1)$, $F(x)=\frac{-1}{\pi}\left(\frac{1}{5} \cos \left(\frac{x \pi}{2}\right)+\frac{1}{3} \cos (x \pi+1)\right)$, with roots $\left(\alpha_{1}, \alpha_{2}, \alpha_{3}, \alpha_{4}\right)=$ $(0.7929,1.6190,2.6251,3.7222), \mu=0.0005, \gamma=0.005$

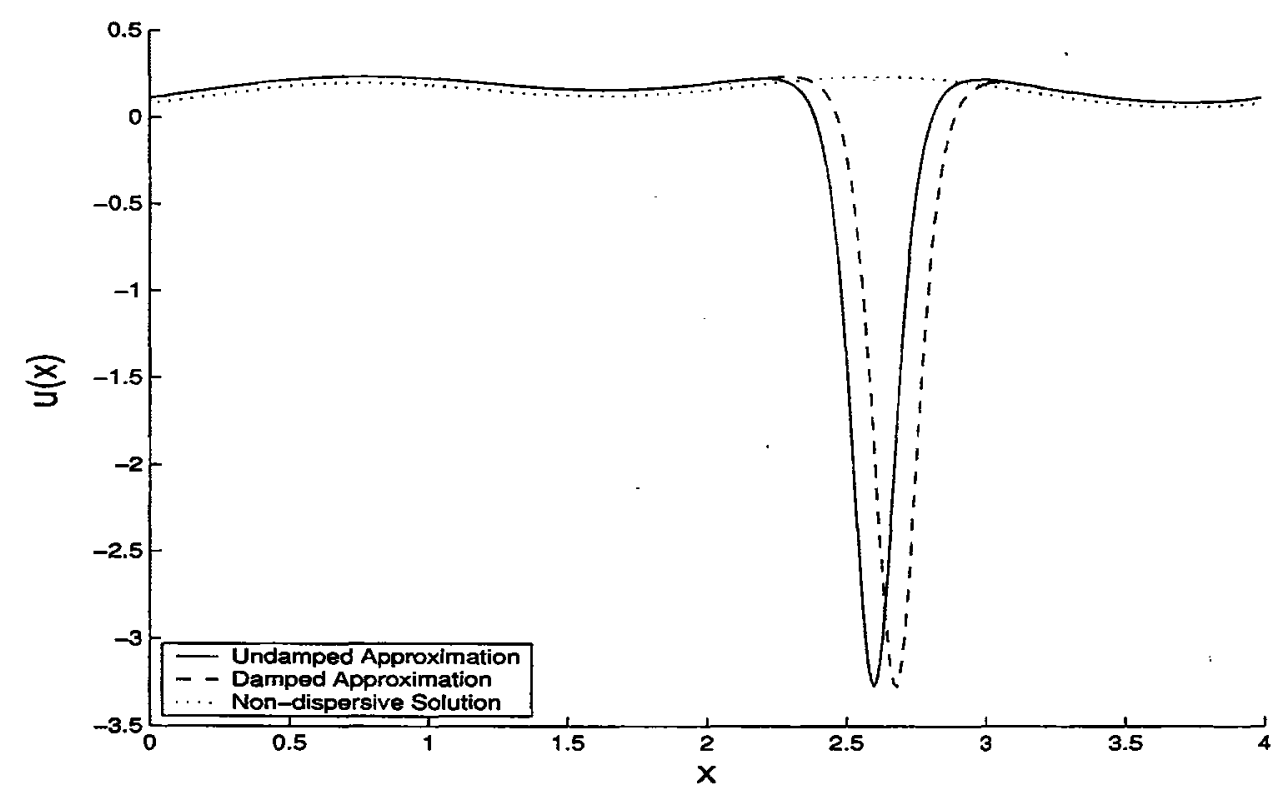

Figure 7: comparison of the approximate solution for $\mu=0$ and $\mu=0.0005$ with $f(x)=\frac{1}{10} \sin \left(\frac{x \pi}{2}\right)+\frac{1}{3} \sin (x \pi+1), \gamma=0.005, \Delta=1.3668$ 


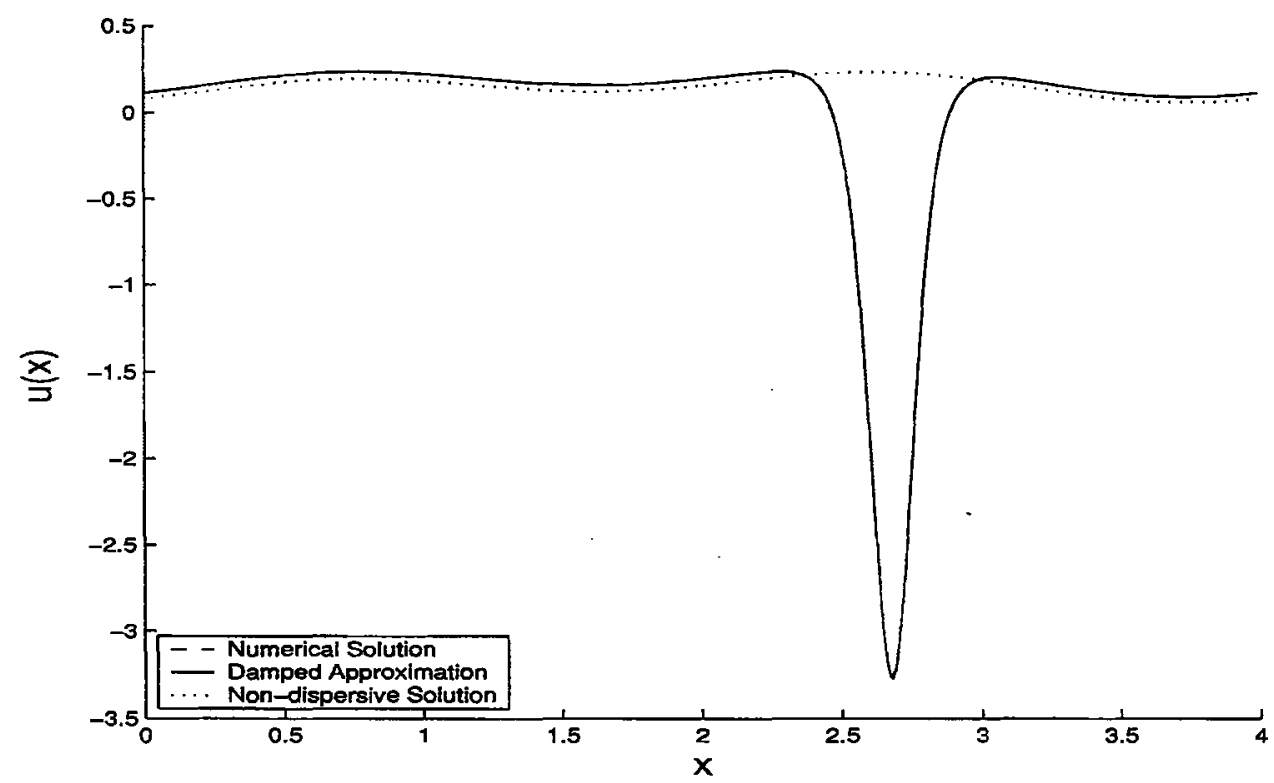

Figure 8: comparison of the numerical solution and leading order damped approximate solution for $f(x)=\frac{1}{10} \sin \left(\frac{x \pi}{2}\right)+\frac{1}{3} \sin (x \pi+1), \mu=0.0005, \gamma=$ $0.005, \Delta=1.3668$

approximation is consistent with the numerical solution, as shown by Figure 8.

Next, the parametric continuation of the solutions is examined. This yields complete bifurcation diagrams, which illustrate the sequence of steady solutions which arise through variation of the detuning parameter.

\subsection{Parametric Continuation}

This section examines parametric continuations of solutions where the forcing corresponds to non-dispersive solutions with a single maximum and minimum, and various qualitatively distinct cases with multiple maxima and 
minima.

\subsubsection{Non-Dispersive Solutions with a Single Maximum and Mini- mum}

As discussed in Section 2.3, for $\mu$ small and positive, peaks are generated at the root of $h(x)$, which is near the global minimum of the non-dispersive solution. Through the variation of $\Delta$, the peaks move to the left and deposit themselves around the adjacent maximum.

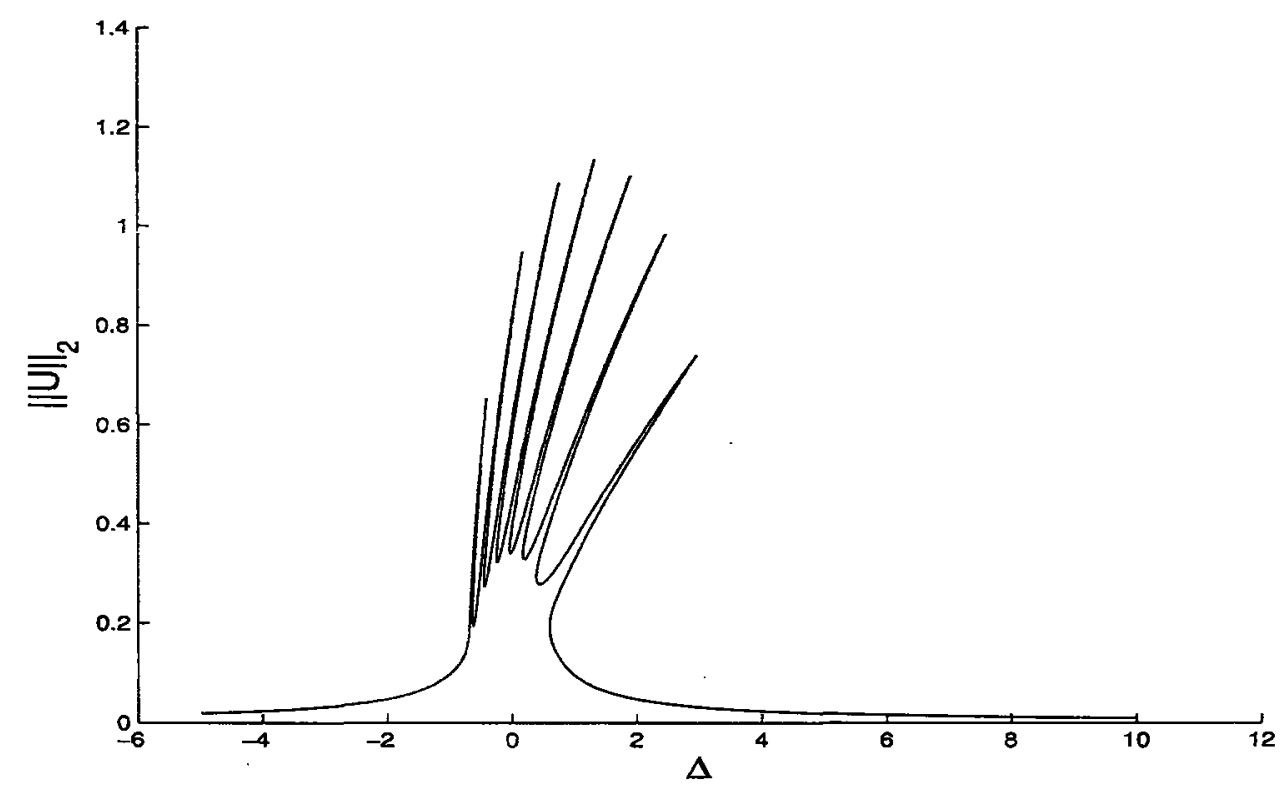

Figure 9: sample bifurcation diagram for $\mu=0.0005$ and $\gamma=0.005$

The bifurcation diagram, Figure 9, shows the parametric continuation of a sample single mode forcing by varying $\Delta$, starting with positive values. The fingers all originate at values of $\Delta$ within the resonant band. Moving from the right, each finger corresponds to the creation of a new peak. At the base of each finger $\|u\|_{2}$ is relatively small, which corresponds to a relatively small 
value of $c$. By (2.32) and as $c \rightarrow c_{\text {min }}$, at some point $x_{i}$ in the solution a new peak is created. Following the bifurcation diagram, $\Delta$ increases, and as will be discussed in Section 4, continues to increase until $c \rightarrow c_{\max }$. After the value of $\Delta$ corresponding to $c_{\max }$ is reached, $\Delta$ begins to decrease. When $\Delta$ returns to the resonant band, $c \rightarrow c_{\text {min }}$. This corresponds to the point where the first peak reaches another root of $h(x)$ (ie. the peak is positioned near a maximum), and by (2.32) also necessitates the creation of another peak. Each finger begins at a unique $\Delta$ because the solution $u$ will have changed with respect to solutions of the previous finger. This in turn affects the equation for $c$ in terms of $\Delta,(2.13)$.

Consider the forcing for the single maximum/minimum case

$$
f(x)=\frac{-3}{10} \cos \left(\frac{x \pi}{2}\right) .
$$

Let $\gamma=0.005$ and $\mu=0.0005$, and the value of $c_{\min }=0.4721$. This yields the roots of $h(x)$

$$
\left(\alpha_{1}, \alpha_{2}\right) \doteq(1.0000,3.1592) .
$$

By the discussion in Section 2.3, the slope at $\alpha_{1}$ is positive, so it corresponds to the location of the peak formation. To visualize the pattern the peaks form through the variation of $\Delta$, the position of each peak is recorded at every leading order approximation. A peak is identified as such if its amplitude is twice that of the global minimum of the non-dispersive solution. The peak pattern diagram shows the location of the peaks through the variation of $\Delta$. Figure 10 clearly depicts the peaks being created near the minimum and moving towards the maximum of the non-dispersive solution. As the first 


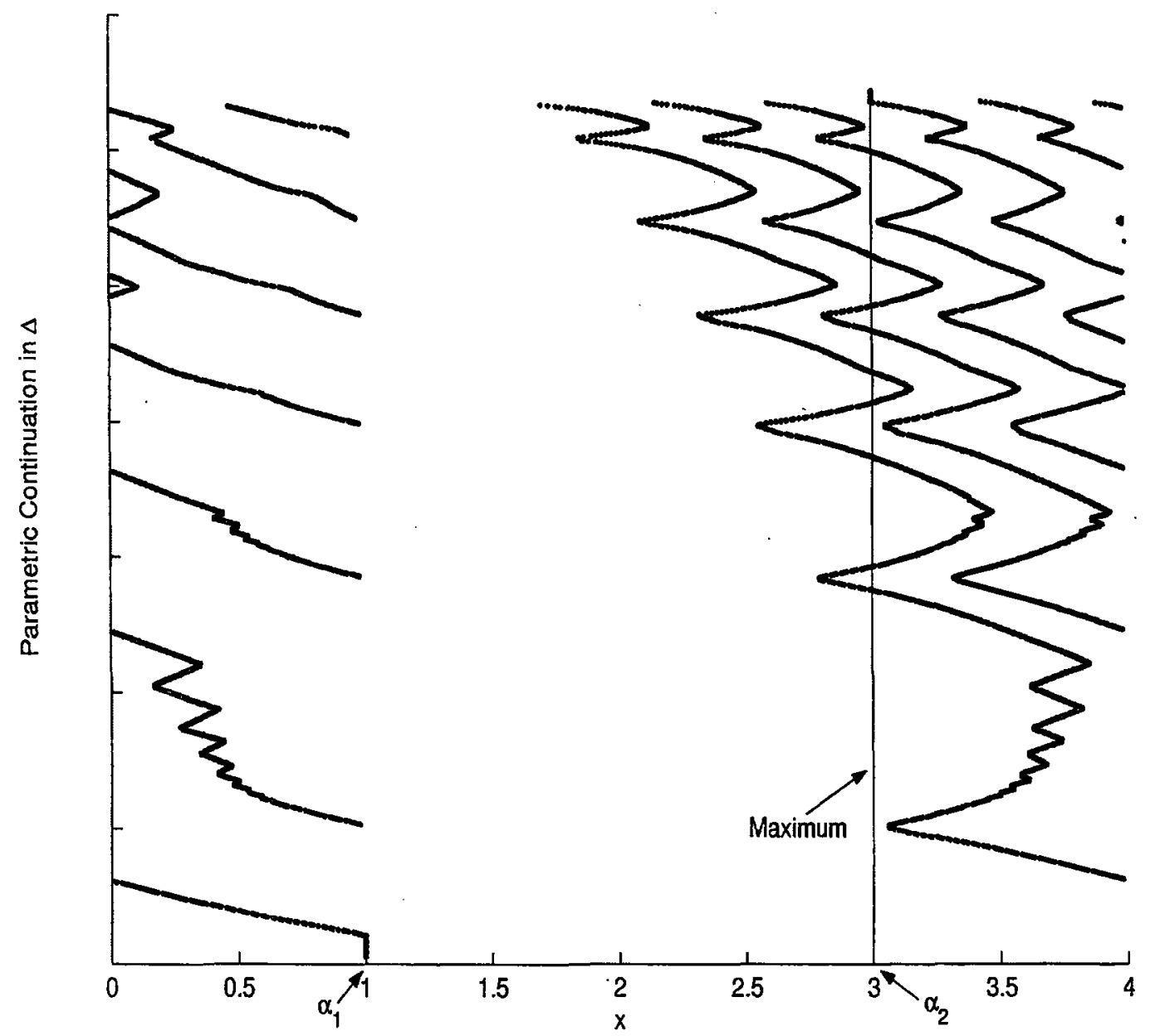

Figure 10: peak pattern diagram for $f(x)=\frac{-3}{10} \cos \left(\frac{x \pi}{2}\right), \mu=0.0005, \gamma=0.005$ peak reaches the vicinity of the maximum, another peak is generated at the minimum.

Now that the parametric continuation of the single mode forcing has been shown, more general forcings may be considered. 


\subsubsection{Non-Dispersive Solutions with Multiple Extrema and One Global Minimum}

Consider the more general forcing which yields a non-dispersive solution with only one global minimum. Through the parametric continuation of the detuning parameter, while there are relatively few peaks in the numerical solutions, the behaviour of a multiple minima/maxima forcing is qualitatively the same as that of the single mode case. However, the pattern the peaks follow is complicated when additional peaks are generated. To examine the behaviour of this class of forcing, they are further broken down into several cases and special cases.

CASE I is classified as a forcing which yields a non-dispersive solution with one global minimum, and one global maximum to its immediate left. Consider the forcing (seen in Figure 11)

$$
f(x)=\frac{1}{10} \sin \left(\frac{x \pi}{2}\right)+\frac{1}{3} \sin (x \pi+1),
$$

which yields a non-dispersive solution with two maxima and two minima, where the global maximum is to the immediate left of the global minimum. Again, $\gamma=.005$ and $\mu=.0005$. Using (2.8) $c_{\min }=0.1629$, and yields the roots of $h(x)$ as

$$
\left(\alpha_{1}, \alpha_{2}, \alpha_{3}, \alpha_{4}\right) \doteq(0.7935,1.6195,2.6251,3.7221)
$$

The root corresponding to the location of peak formation is $\alpha_{4}$. As the peak moves, it is deposited near $\alpha_{3}$ which is near the global maximum of the nondispersive solution. 


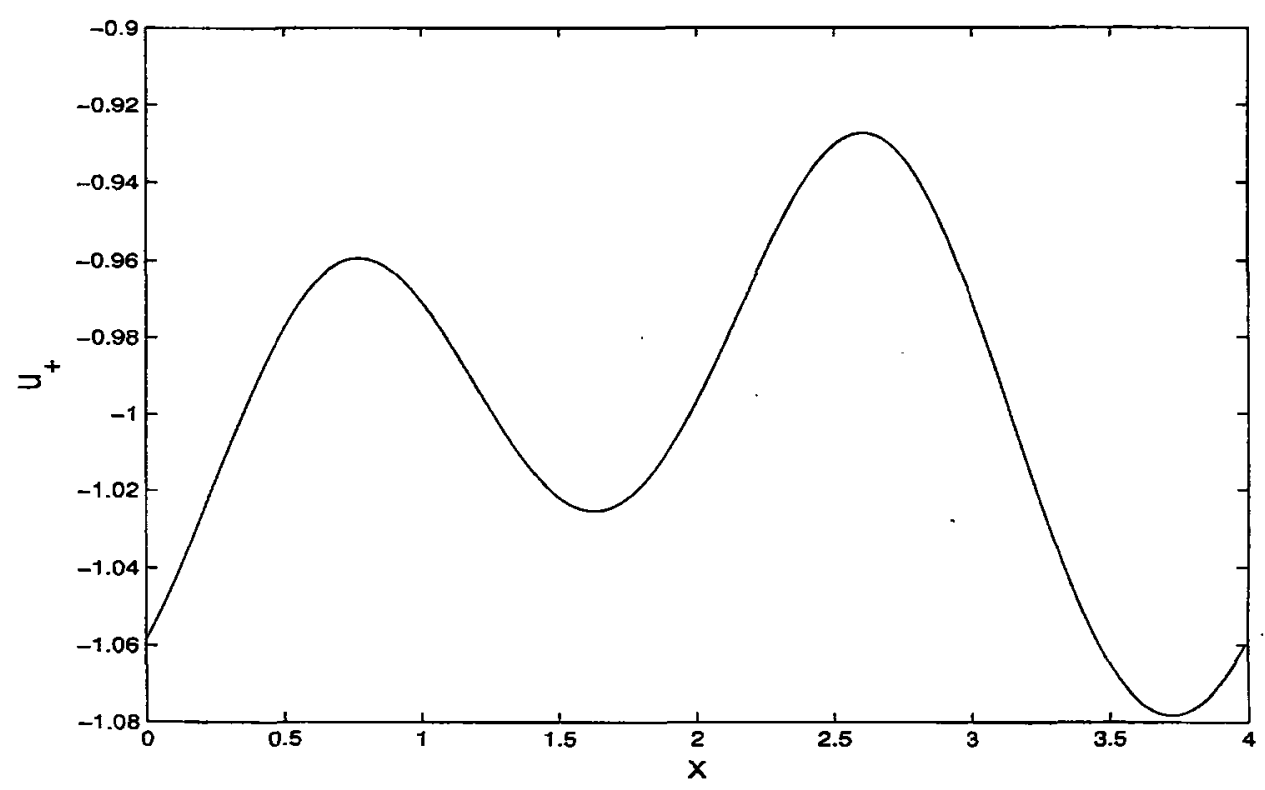

Figure 11: non-dispersive solution corresponding to $f(x)=\frac{1}{10} \sin \left(\frac{x \pi}{2}\right)+$ $\frac{1}{3} \sin (x \pi+1)$

In the peak pattern diagram, Figure 12 , it is possible to track the generation of new peaks, their motion towards the adjacent maximum, and overcrowding, the shifting of peaks to the next maximum. Overcrowding occurs when the number of peaks at the maximum adjacent to the global minimum reaches a critical value. When the number of peaks exceeds the critical value, the leftmost peak shifts to the next maximum. There is an effective potential barrier from the other local minimum which prevents the peak from initially depositing itself at other maxima, but due to overcrowding this potential barrier is overcome by the first peak produced. As more peaks are generated, more peaks shift over to the second maximum.

In this problem, it was found that overcrowding occurs when more than 3 peaks are deposited around a given maximum. When this happens, the 


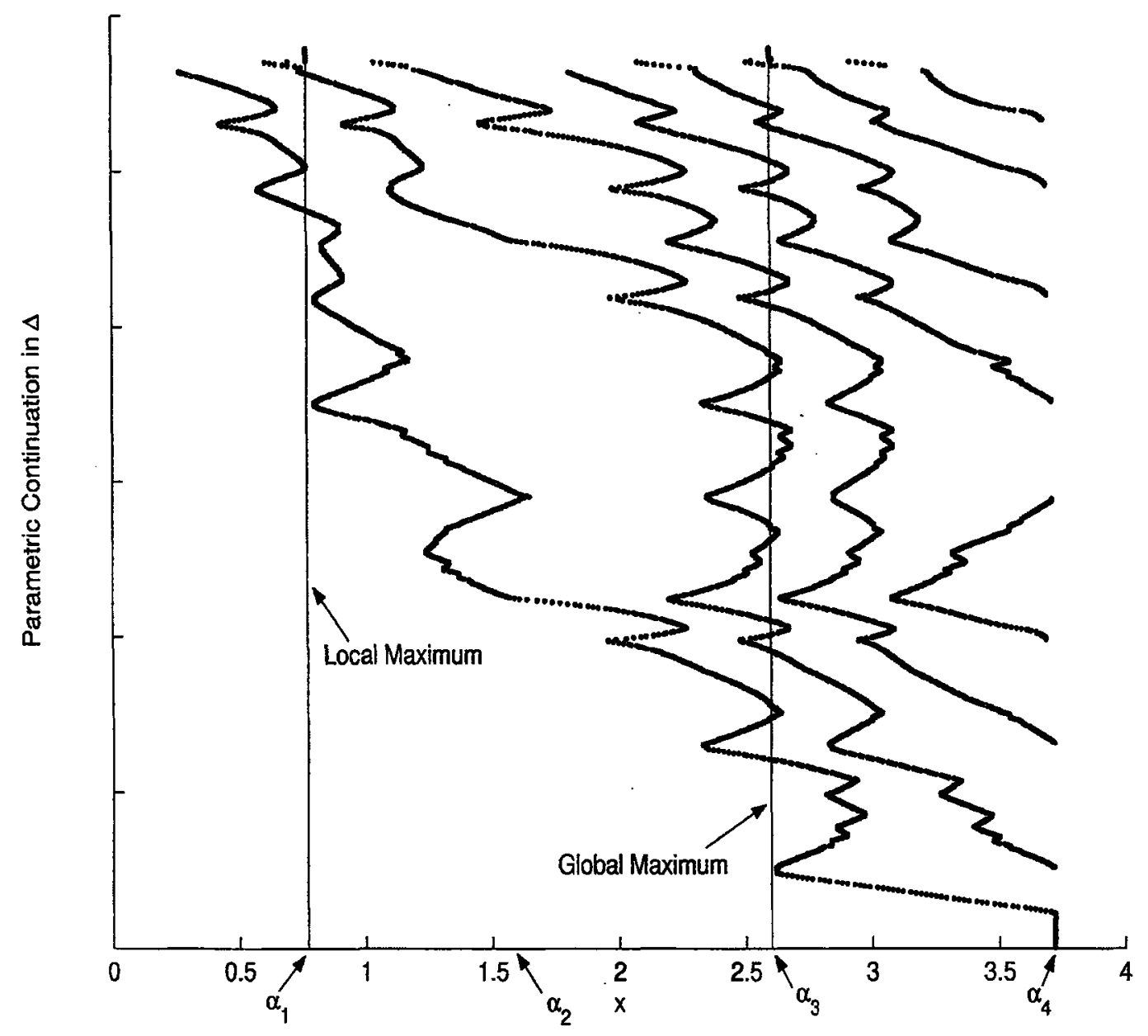

Figure 12: peak pattern diagram for $f(x)=\frac{1}{10} \sin \left(\frac{x \pi}{2}\right)+\frac{1}{3} \sin (x \pi+1), \mu=$ $0.0005, \gamma=0.005$

leftmost peak shifts to the next maximum. The critical value is governed by the width of the peaks, $O(\sqrt{\gamma})$. By changing $\gamma$ for a fixed forcing, it is possible to generate peak pattern diagrams with different critical values of overcrowding. Generating a set of parametric continuations with values of $\gamma$ ranging from $\gamma=0.005$ to $\gamma=0.025$ yields a range of critical values of overcrowding. 
Since the peak widths are $O(\sqrt{\gamma})$, and thus greater values of $\gamma$ yield lower critical values, an inverse relation is expected between the critical value of overcrowding and peak width. The function relating $\gamma$ and critical values of crowding is expected to be $\frac{\tilde{\xi}}{\sqrt{\gamma}}$, where $\tilde{\xi}$ is a constant which depends on the forcing. For this particular forcing, applying a least squares approximation to the critical values of overcrowding and their related values of $\gamma$ yields a similar function, $\tilde{\xi} \gamma^{\rho}$, where $\rho=-0.52$ (the slope of the best fit line) and $\tilde{\xi}=1.87$ (the intercept of the best fit line). Thus, the least squares approximation yields the functional form $\tilde{\xi} \gamma^{\rho} \approx \frac{\bar{\xi}}{\sqrt{\gamma}}$, as expected.

The peak pattern diagram for this forcing, Figure 12, shows that the majority of the peaks deposit at the global maximum. When overcrowding occurs, the leftmost peak shifts to the other (local) maximum. There is a bias for peaks to deposit at the global maximum, which in this case is to the immediate left of the global minimum. In order to understand the dependence of $\tilde{\xi}$ on the forcing, another forcing is considered.

CASE II is classified as a forcing which yields a non-dispersive solution, such as Figure 13, where the global maximum is not immediately to the left of the global minimum. This forcing yields a qualitatively different peak pattern diagram, Figure 14, in comparison to the previous forcing. The critical value of overcrowding for this case is approximately 2.5 .

The critical values of overcrowding can be affected by two factors. The first is that in the non-dispersive solutions the potential barrier for this problem, Figure 15, is less than in the previous case, Figure 16. Due to a smaller potential barrier, the peaks will shift to the next maximum more readily. As 


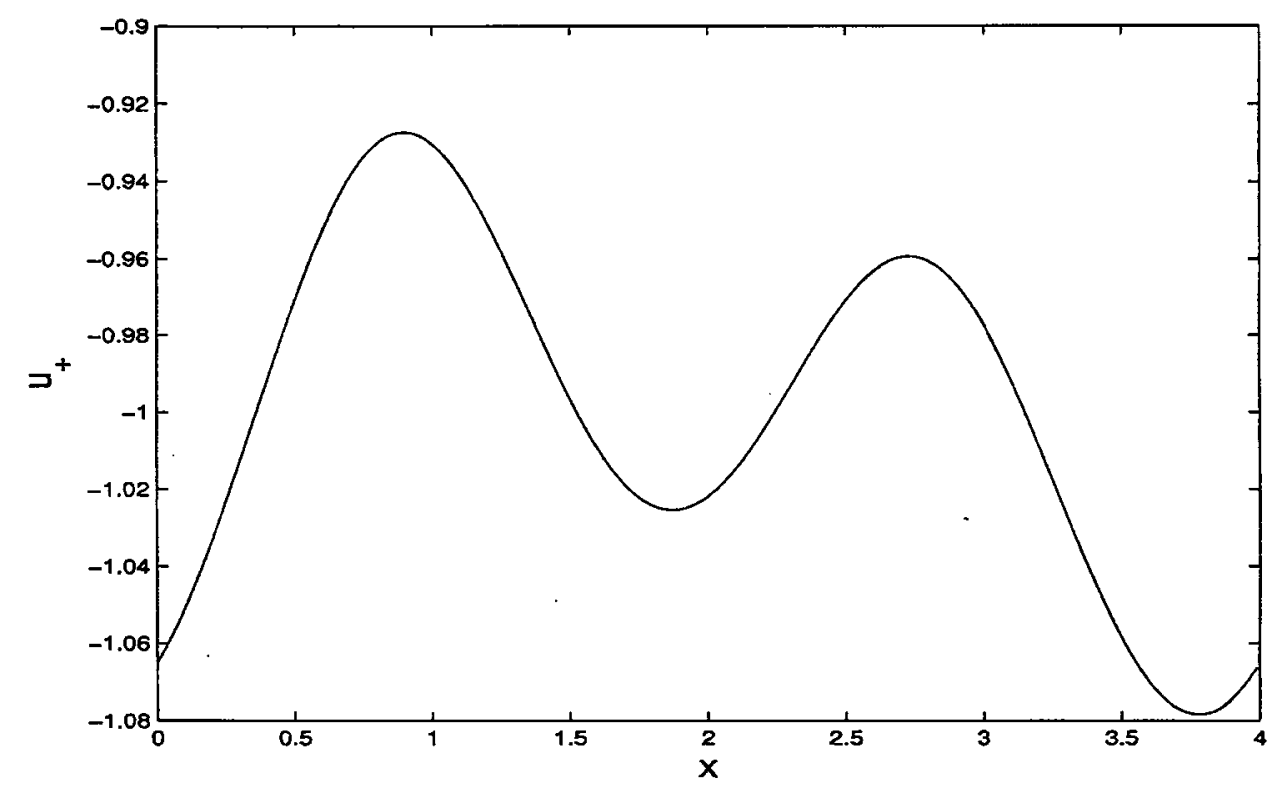

Figure 13: non-dispersive solution corresponding to the forcing in the second case

a result the critical value of overcrowding is less for this forcing. The second factor is the significance of the position of the global maximum.

To examine this factor, consider the special case forcing which yields a nondispersive solution with two equal global maxima, one global minimum, and one local minimum, such as shown in Figure 17. The peak pattern diagram for this special case, Figure 18, is almost identical to the peak pattern diagram for the forcing from case 2, Figure 14, and also indicates a critical value of overcrowding of approximately 2.5. The effective potential barrier for the special case, Figure 19, is similar to that of the second case, and less than in the first case, and thus yields the same critical value of overcrowding as in the second case. This indicates that the position of the global maximum is not a significant factor affecting the critical value of overcrowding. 


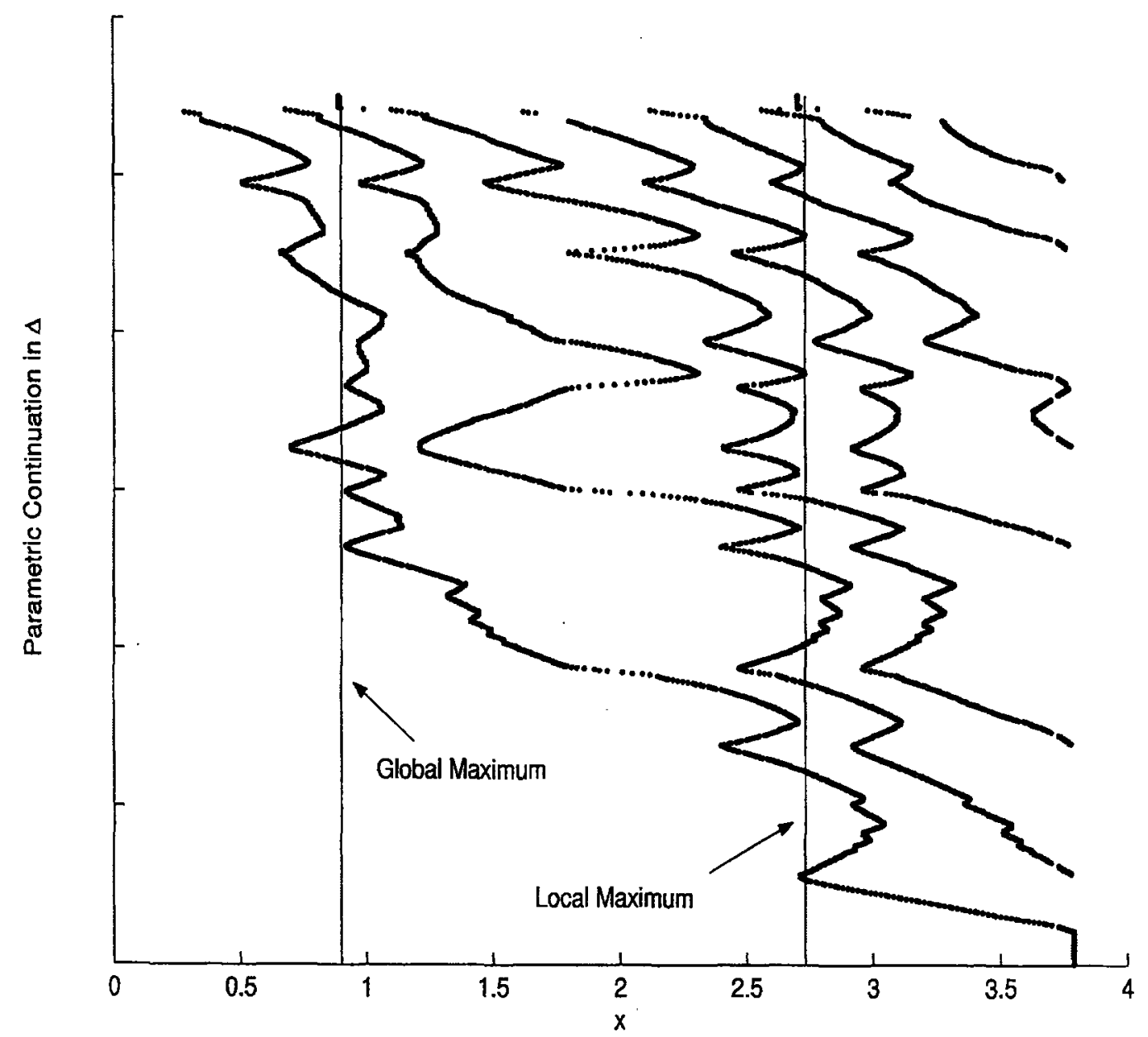

Figure 14: peak pattern diagram for the second case with $\mu=0.0005$ and $\gamma=0.005$ 


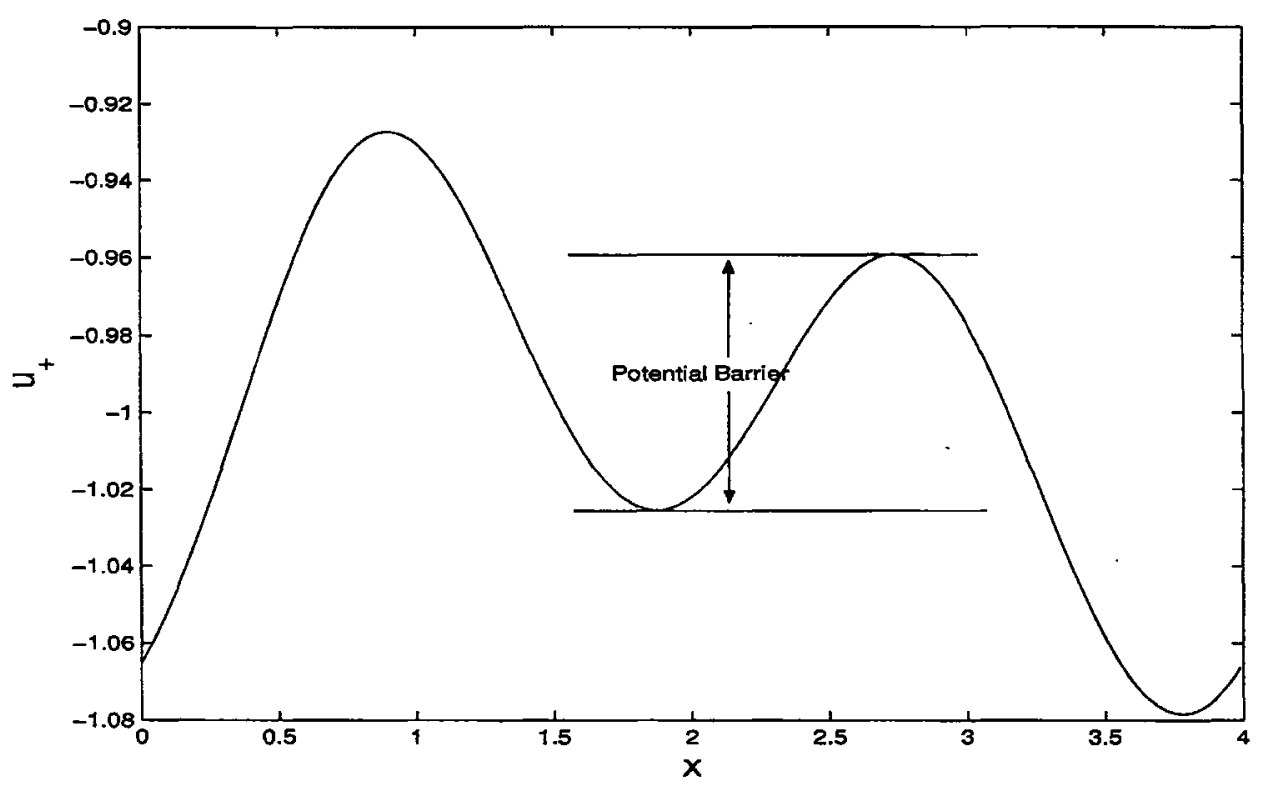

Figure 15: potential barrier of the non-dispersive solution for the second case

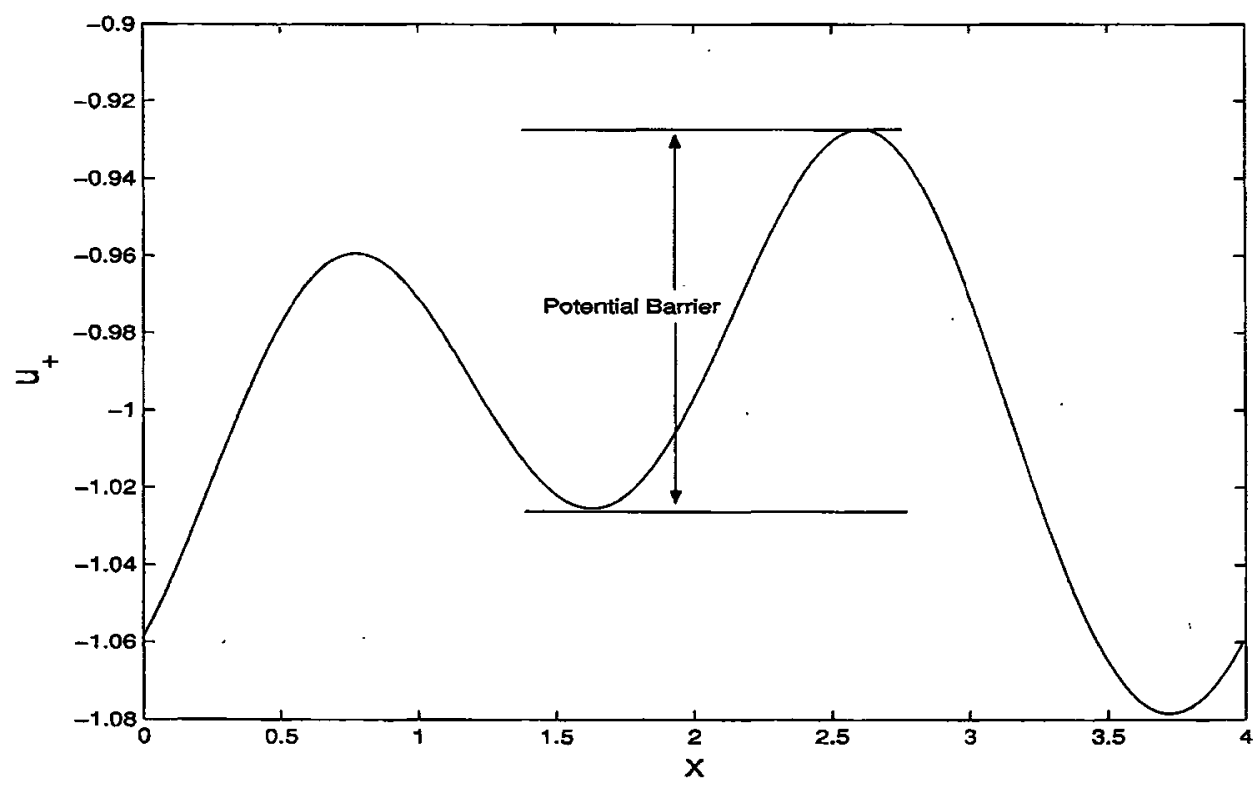

Figure 16: potential barrier of the non-dispersive solution for the first case 


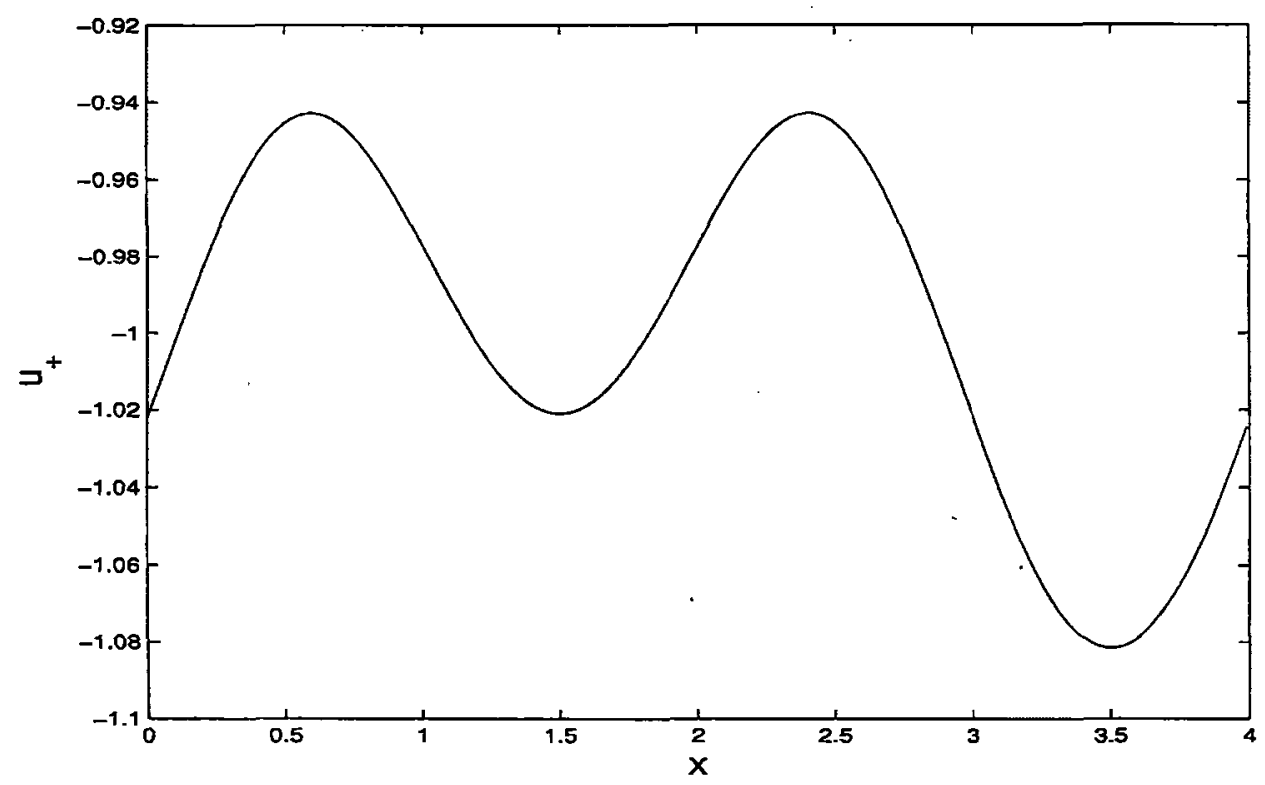

Figure 17: non-dispersive solution with two equal global maxima corresponding to the first special case

For the second case and the special case, varying $\gamma$ changes the critical value of overcrowding as it does for the first case. For $\gamma=0.005$ the critical values of overcrowding are different from those of the first case, so the curve $\tilde{\xi} \gamma^{\rho}$ would have different values of $\tilde{\xi}$. The function relating peak width and critical value of overcrowding would still be $0\left(\gamma^{\rho}\right)$, but the value of $\tilde{\xi}$ would be different because of the dependence on the forcing.

The other effect of peak width is the saturation of the domain. At a certain point, so many peaks are present that there is no longer any clear qualitative pattern of peak distribution. Again, the number of peaks for saturation to occur is dependent on peak width. The function that relates the number of peaks and $\gamma$ is again expected to take the functional form $\tilde{\xi} \gamma^{\rho} \approx \frac{\tilde{\xi}}{\sqrt{\gamma}}$. The least squares approximation from a series of parametric continuations with 


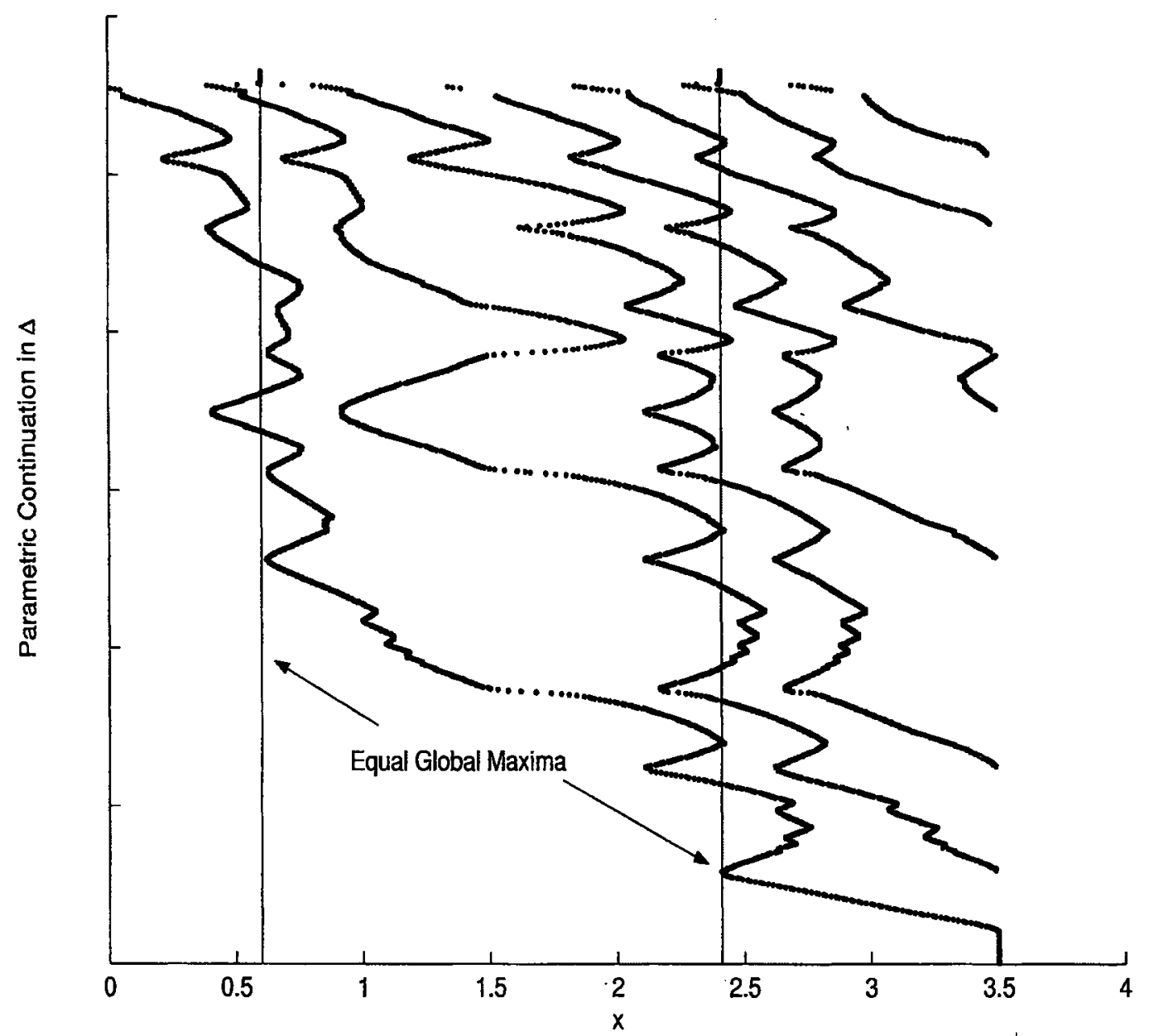

Figure 18: peak pattern diagram for the first special case with $\mu=0.0005$ and $\gamma=0.005$

varied $\gamma$ and the forcing from the first case yielded $\rho=-0.40$ and $\tilde{\xi}=2.62$. For $\gamma=0.005$ the peak pattern diagram, Figure 12, indicates that saturation occurred after the seventh peak was generated. As seen in Figures 14 and 18, for $\gamma=0.005$ the value for the saturation of the domain for the second case and the special case is also 7. Unlike overcrowding, the critical value of domain saturation is not dependent on the forcing, so the function is $\xi \gamma^{\rho}$ where $\xi$ is dependent only on $\gamma$. 


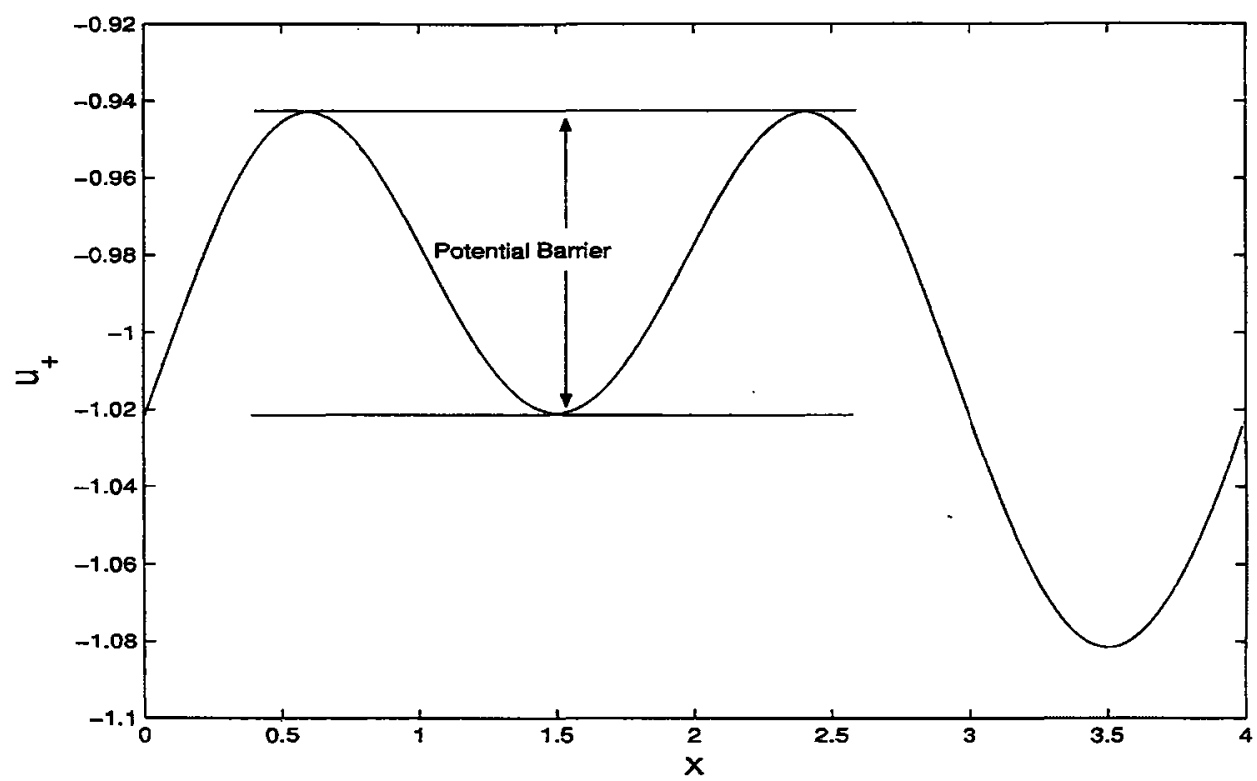

Figure 19: potential barrier of the non-dispersive solution for the first special case

Now that the class of forcing which yields non-dispersive solutions with one global minimum has been investigated, the class of forcing which yields a non-dispersive solution with multiple global minima is considered.

\subsubsection{Non-Dispersive Solutions with Multiple Extrema and Multi- ple Global Minima}

Another special case occurs when a forcing yields a non-dispersive solution with multiple global minima on a single period. Numerically this is difficult to investigate, but following the same steps as in Section 2.3 , it can be shown that it is possible to have peaks being created at multiple points if the forcing yields a non-dispersive solution with multiple global minima. Assuming the condition (2.8) is maintained, multiple cusps will form as $c \rightarrow c_{\min }$. Every value of $x_{i}$ 
which satisfies (2.32) corresponds to the location of a global minimum of the non-dispersive solution.

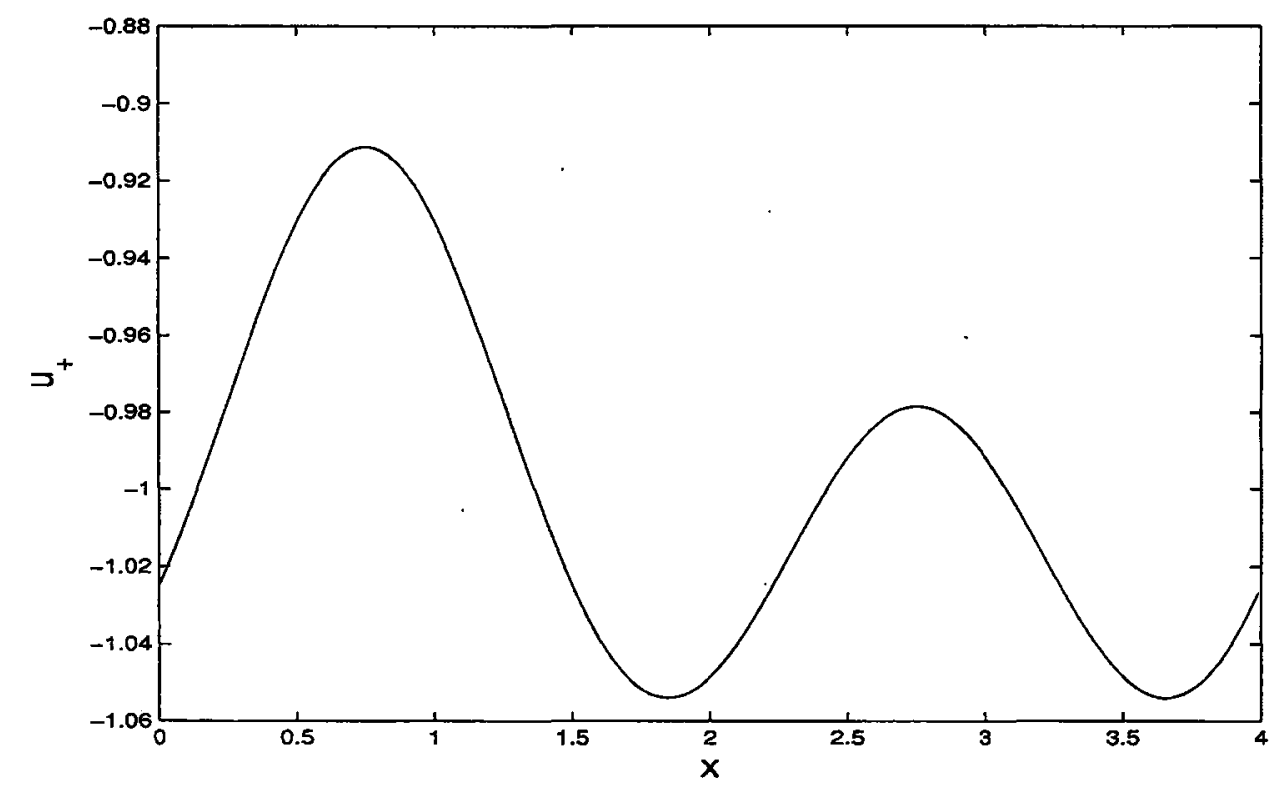

Figure 20: non-dispersive solution corresponding to $f(x)=\frac{1}{10} \sin \left(\frac{x \pi}{2}\right)+$ $\frac{1}{3} \sin (x \pi)$

Consider the forcing which yields a non-dispersive solution with two equal global minima, such as Figure 20. Two cusps will form at the two values of $x_{i}$ corresponding to (2.32). For the solution to remain smooth and differentiable, two peaks will form simultaneously at each global minimum. In order to study this special case with two equal global minima, consider the forcing

$$
f(x)=\frac{1}{10} \sin \left(\frac{x \pi}{2}\right)-\frac{1}{3} \sin (x \pi+\delta)
$$

which yields a non-dispersive solution with two maxima and two minima, with an added parameter $\delta$. As $\delta \rightarrow 0$, the problem becomes the special case where there exist two equal global minima in the non-dispersive solution. Letting 


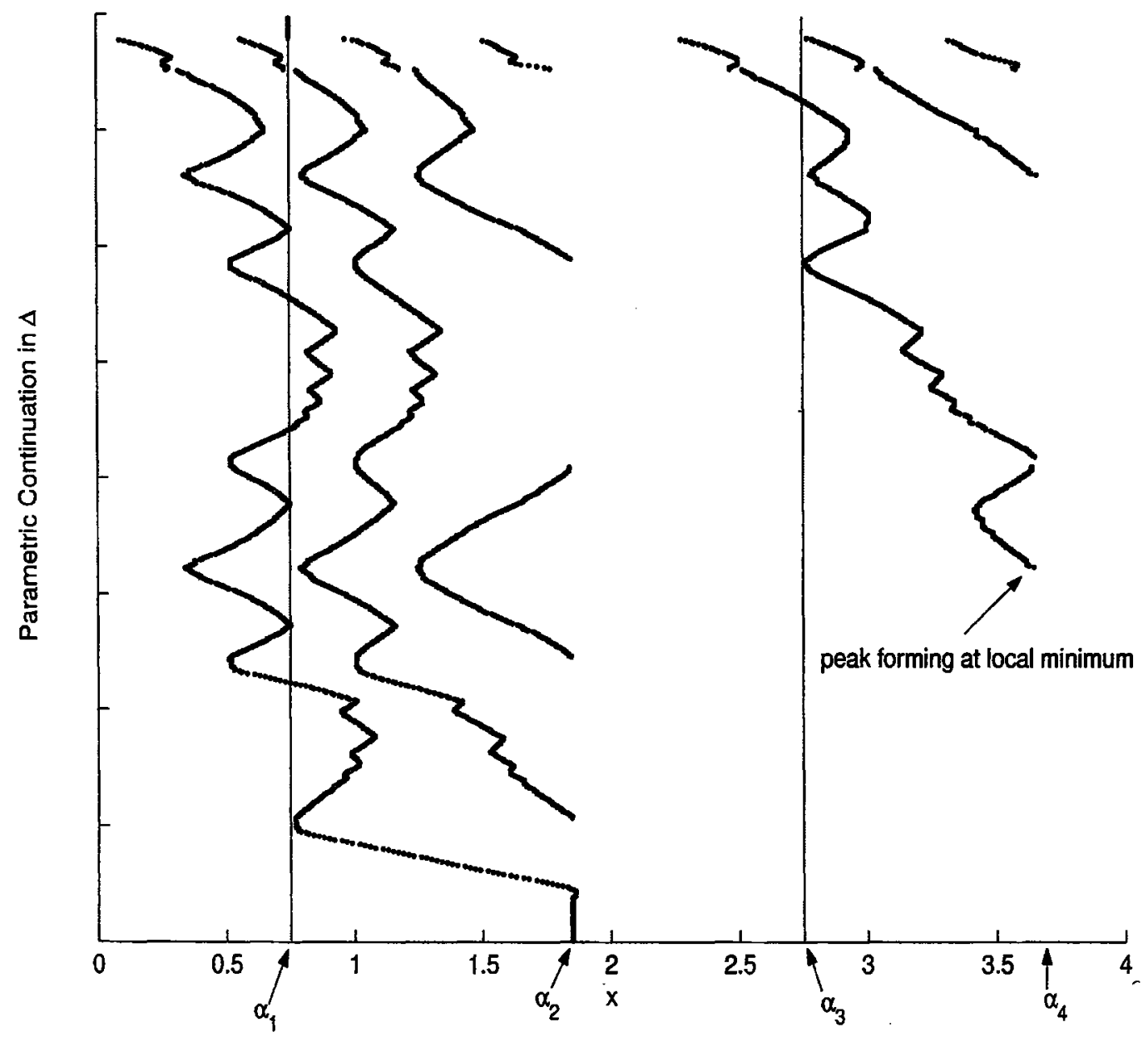

Figure 21: peak pattern diagram for $f(x)=\frac{1}{10} \sin \left(\frac{x \pi}{2}\right)+\frac{1}{3} \sin (x \pi-0.01)$, $\mu=0.0005, \gamma=0.005$

$\delta=-0.01$, then the roots of $h(x)$ are

$$
\left(\alpha_{1}, \alpha_{2}, \alpha_{3}, \alpha_{4}\right) \doteq(0.7785,1.8456,2.7846,3.6636)
$$

The global minimum corresponds to $\alpha_{2}$, and the local minimum corresponds to $\alpha_{4}$. Conversely, for $\delta>0$, the global minimum would correspond to $\alpha_{4}$, and the local minimum to $\alpha_{2}$. As seen in Figure 21, the first three peaks form at $\alpha_{2}$. However, the fourth peak forms at $\alpha_{4}$. This shows that 
peaks may form at either of the global minima and suggests that peaks will form at both global minima if $\delta=0$. Unfortunately, such a case is difficult to solve numerically, but this leads to the conclusion that for the general nondispersive solution with multiple global minima, peaks will form at all roots of $h(x)$ corresponding to global minima of the non-dispersive solution.

Now that the behaviour of the solutions through variation of the detuning parameter is better understood, stability of the solutions, and regions of solution stability of the parametric continuation are examined. 


\section{Stability of Steady Solutions}

This section examines the regions of stability of the general matched, uniformly valid leading order solutions, and the numerical solutions. First regions of stability and the points of change in stability are described with respect to the regions surrounding the extrema in the non-dispersive solution. Next, regions of solution stability for the single mode forcing are identified in the bifurcation diagram and the corresponding peak pattern diagram. These results are extended from the single mode forcing to the general forcing, and are compared to the numerical results produced in both the bifurcation diagram and the peak pattern diagrams for a selected forcing.

\subsection{Regions of Stability for the General Non-Dispersive Solution}

As discussed in Sections 2.3 and 3.2.3, peaks are generated at the global minima of the non-dispersive solution. As $\Delta$ is increased, the amplitude of the peak increases and the peak begins to move to the left (for $\mu$ small and positive). As discussed by Amundsen, Cox, and Mortell [4], the region surrounding a minimum of the non-dispersive solution corresponds to a region of instability. In the case that this region is centred about a global minimum of the non-dispersive solution, it also corresponds to the location of peak creation as the solution is continuously varied across the resonant band. When peaks are created, only a single peak is required to maintain smoothness and differentiability within such a region. The corresponding solution within the 
dispersive layer surrounding a minimum is a single peak, more precisely, the peak corresponds to an unbounded solution because the layer is within a region of instability .

Conversely, the region surrounding a maximum corresponds to a region of stability. The solution with the stable region may contain either form of $\Phi(x),(2.30) ;$ a single peak (bounded solution), or multiple peaks (bounded oscillatory solution).

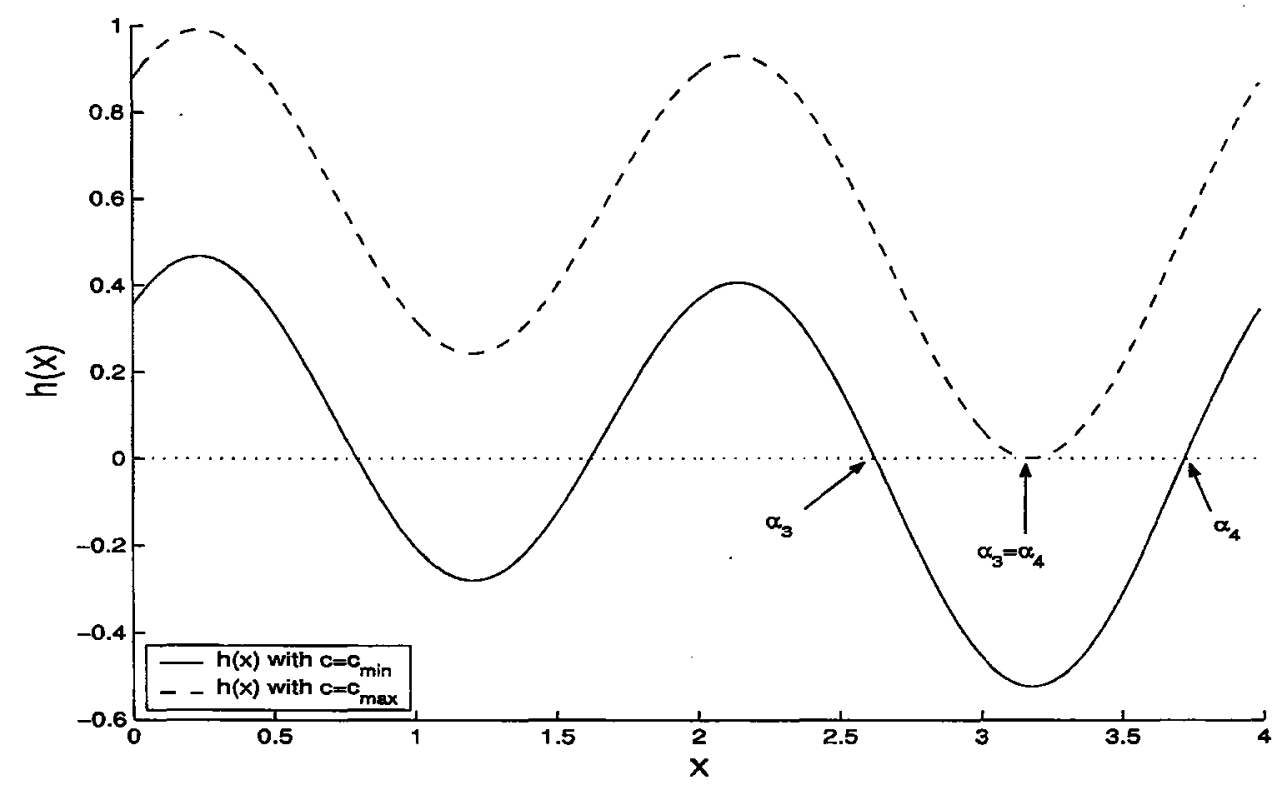

Figure 22: sample $h(x)$ for $c=c_{\min }$ and $c=c_{\text {max }}$, with $\mu=0.0005, \gamma=0.005$

The transition between stable and unstable solutions occurs when two roots of $h(x)$ converge and corresponds to a saddle-node bifurcation. As $c \rightarrow c_{\max }$, the function $h(x)$ shifts upwards in the range and reaches a point where two roots become one, as seen in Figure 22. Considering the known expression

$$
h(x)=\frac{\mu}{\gamma}(F+c)+\frac{5}{4} f,
$$


setting it equal to zero, and solving for $c$ would yield

$$
c=-\frac{5 \gamma}{4 \mu} f-F
$$

For any given forcing $f(x)$, the maximum of $c$ yields

$$
c_{\max }=\max _{x \in[0,2 L]}\left(-\frac{5 \gamma}{4 \mu} f-F\right) .
$$

Using the known value of $c_{\max }$ in $h(x)$, its root, $\alpha^{*}$, corresponds to the peak location for change of solution stability.

If any peak is located within an unstable region of the non-dispersive solution, it renders the entire solution unstable.

Next, using numerical methods stable and unstable solutions will be further examined through the parametric continuation of $\Delta$.

\subsection{Solution Stability for the Single Mode Forcing}

The regions of solution stability and the points of change in solution stability can be seen in a bifurcation diagram. Consider the single mode forcing seen in Section 3.2.1

$$
f(x)=\frac{-3}{10} \cos \left(\frac{x \pi}{2}\right) .
$$

Figure 23 is a bifurcation diagram which shows regions of solution stability. The peaks form as $c \rightarrow c_{\text {min }}$ which corresponds to a value of $\Delta$ within the resonant band by (2.7). After a peak is created $\Delta$ increases and the unstable peak increases in magnitude and moves to the left. As the peak continues to move and grow in magnitude, at a certain point $c$ reaches an upper bound, 


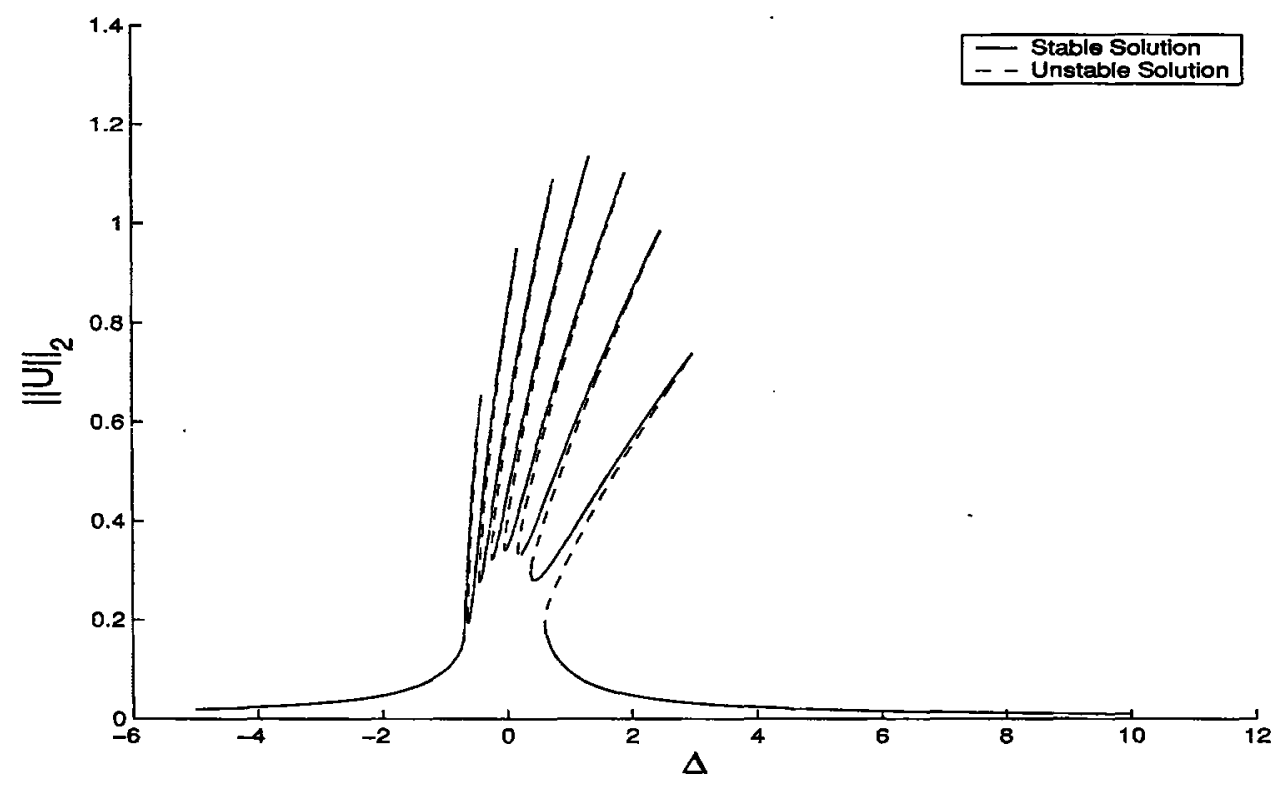

Figure 23: bifurcation diagram indicating regions of stability for $f(x)=$ $\frac{-3}{10} \cos \left(\frac{x \pi}{2}\right), \mu=0.0005, \gamma=0.005$

$c_{\max }$. This corresponds to the peak moving into a stable region, and at this point stability of the solution changes and $\Delta$ begins to decrease. By (4.36), the point of change in stability for the first peak occurs when $c_{\max }=3.7795$, and by (2.13) yields the corresponding value $\Delta=2.9712$. As $\Delta$ decreases the stable peak moves within the region surrounding the maximum. When $c \rightarrow c_{\min }$ another peak is formed at the global minimum. Subsequent values of $\Delta$ corresponding to bifurcations must be determined numerically because of the difficulty associated with the creation of analytic approximations with multiple peaks which can be applied to (2.13).

The point of change in solution stability for the single maximum/minimum forcing can also be seen with respect to the peak location in the peak pattern diagram with stability, Figure 24. The unstable solutions have their peak 


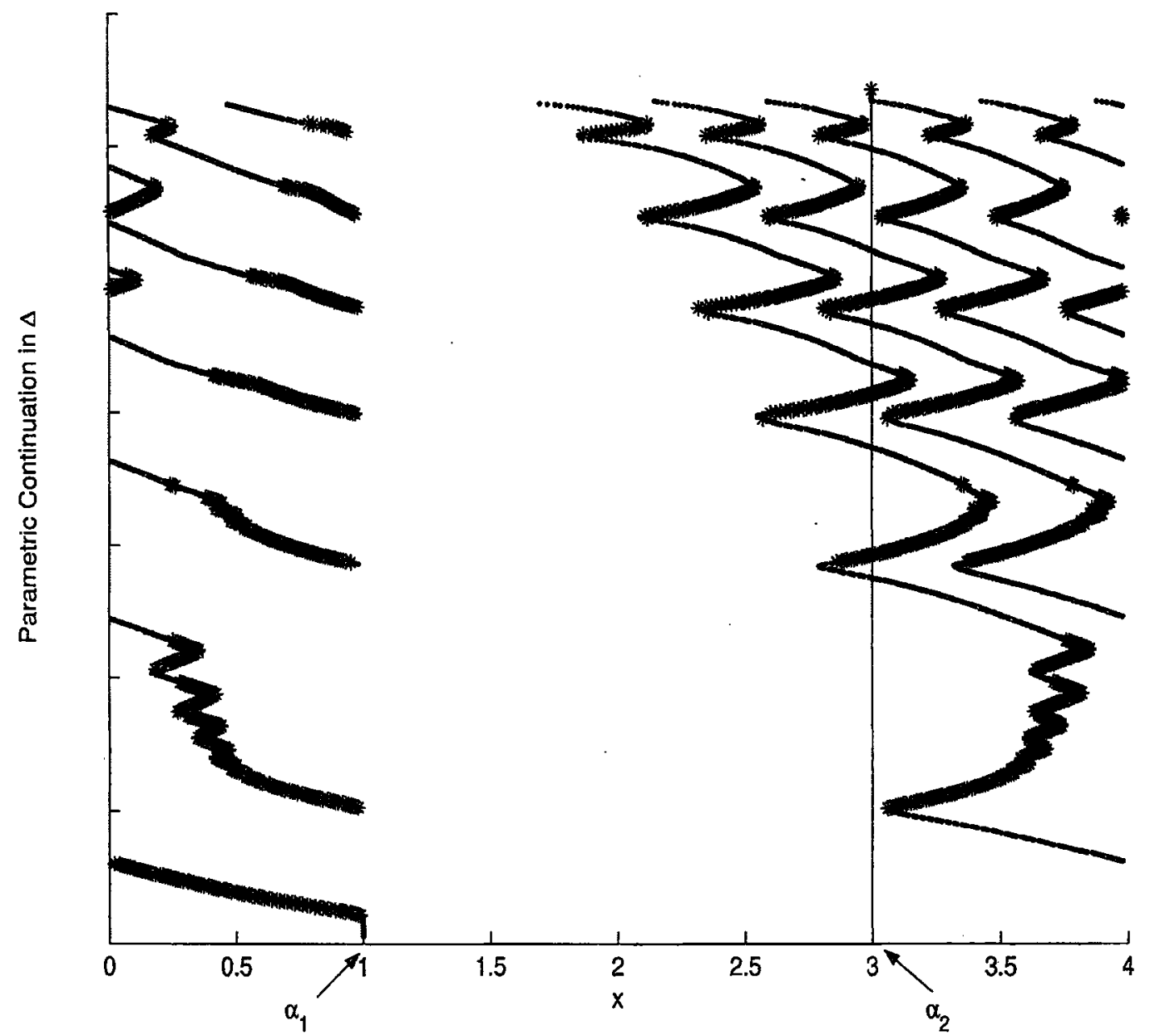

Figure 24: peak pattern diagram with unstable solutions indicated by ' $*$ ' symbols and stable solutions by points, for $f(x)=\frac{-3}{10} \cos \left(\frac{x \pi}{2}\right), \mu=0.0005$, $\gamma=0.005$ 
locations indicated by '*' symbols, and stable solution are indicated by points. For the first peak, the change in stability occurs at $x \doteq 0.06$. By (4.36), the change in solution stability occurs at $\alpha^{*} \doteq 0.08$, which is consistent with the numerical result. The peak pattern diagram also indicates that while at least one of the peaks is located in an unstable region, the entire solution is unstable.

Next, this behaviour is extended to a general forcing, and is compared to the results discussed here.

\subsection{Solution Stability for the General Forcing}

Now that regions of solution stability are qualitatively understood for the single mode forcing, a more general forcing is considered. To further examine the behaviour of the general forcing, recall the forcing in the first case which yields a non-dispersive solution with two maxima and two minima

$$
f(x)=\frac{1}{10} \sin \left(\frac{x \pi}{2}\right)+\frac{1}{3} \sin (x \pi+1)
$$

This forcing yields a bifurcation diagram, Figure 25, which is similar to the bifurcation diagram of the single mode forcing. The diagram describes the same qualitative behaviour as for the single mode forcing. Using (2.7) and (4.36), the point of change in stability for the first peak is $\Delta=5.909$, derived from $c_{\max }=5.3842$.

The peak pattern diagram, Figure 26, shows solution stability in relation to peak location. Using the known value of $c_{\max }$ from (4.36), the change in solution stability of the first peak is expected to occur at $\alpha^{*} \doteq 3.18$. The 


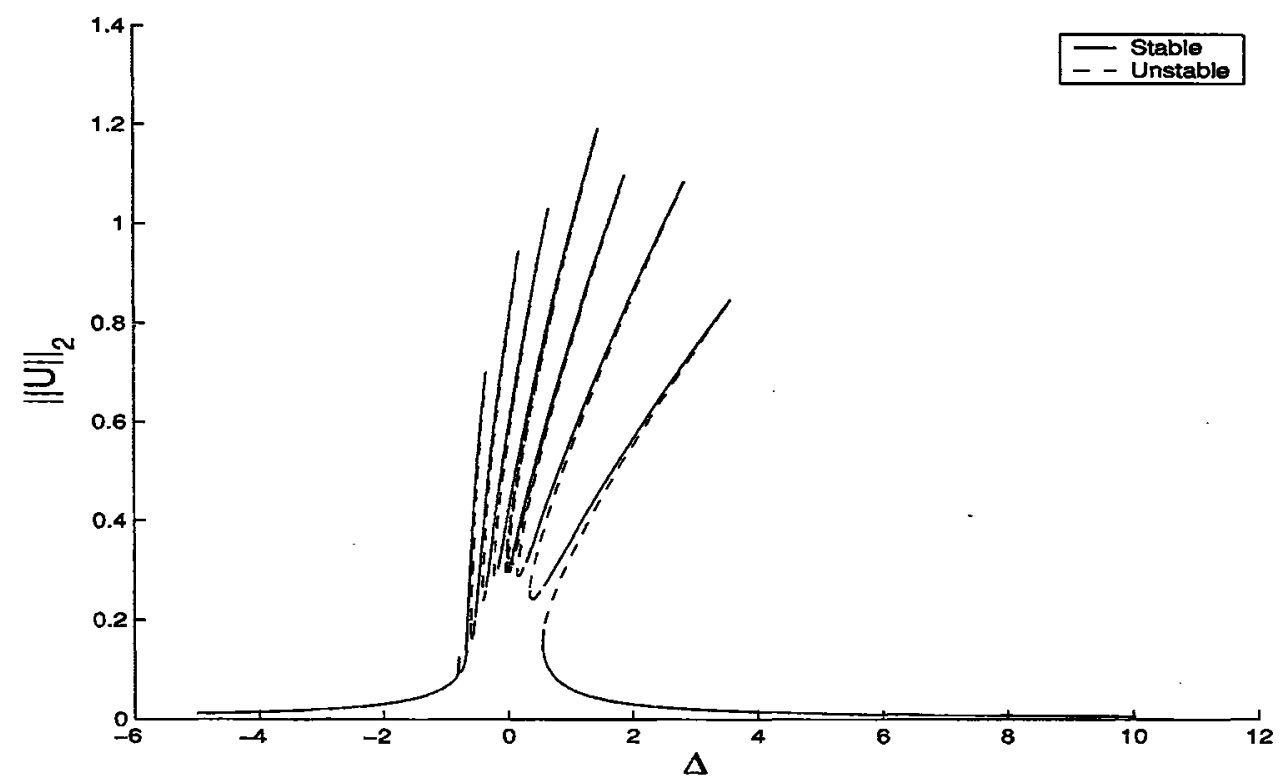

Figure 25: bifurcation diagram indicating regions of stability for $f(x)=$ $\frac{1}{10} \sin \left(\frac{x \pi}{2}\right)+\frac{1}{3} \sin (x \pi+1), \mu=0.0005, \gamma=0.005$

numerical results yield the same value, $x \doteq 3.18$. The behaviour is similar to that of the single mode forcing in that when peaks are generated the entire solution becomes unstable, but is different in the sense that stability is affected when peaks shift due to the effect of peak overcrowding. When a maximum becomes overcrowded and a peak shifts to the next maximum, the solution becomes unstable as it passes through the unstable region corresponding to the intermediate minimum.

The conclusion follows that a general forcing will yield a similar bifurcation diagram with the same qualitative regions of solution stability provided the width of the peaks is much less than the width of the maxima and minima of the non-dispersive solution. It also demonstrates that solutions become unstable whenever one of the peaks is located in the vicinity of a minimum of 
the non-dispersive solution, regardless of whether it is the origin of the peak, or a peak moving through the region due to the effects of overcrowding.

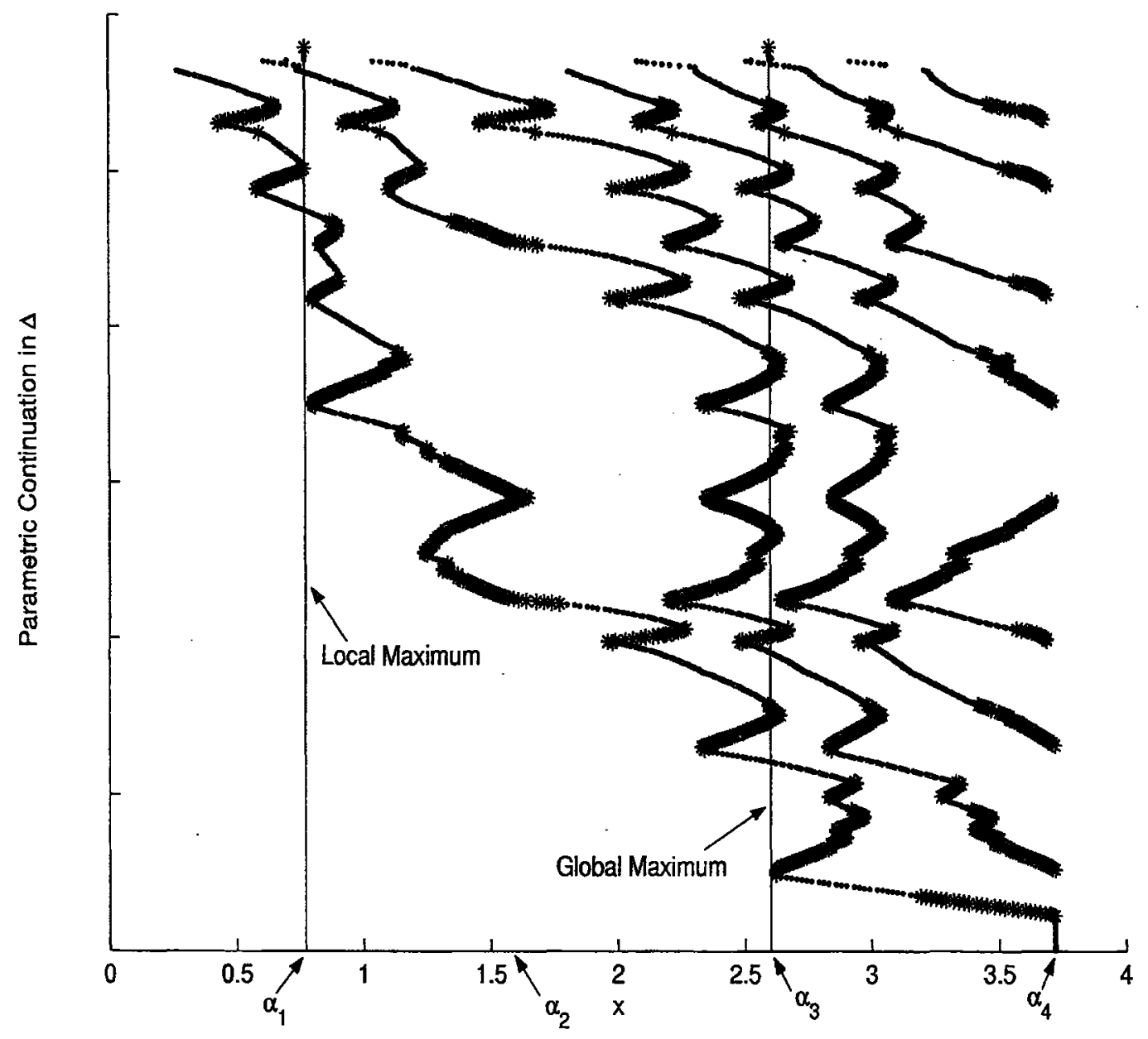

Figure 26: peak pattern diagram with unstable solutions indicated by ' $*$ ' symbols and stable solutions by points, for $f(x)=\frac{1}{10} \sin \left(\frac{x \pi}{2}\right)+\frac{1}{3} \sin (x \pi+1)$, $\mu=0.0005, \gamma=0.005$ 


\section{Conclusion}

The preceding analysis effectively demonstrated analytic and numerical methods being applied to solving the steady state periodically forced $\mathrm{KdV}$ equation with general forcing. The behaviour seen for the single mode forcing was successfully extended to a general forcing, and showed that many behaviours on a local level remained the same. Also, more global occurrences which arose from the fact that the forcing now yielded a non-dispersive solution with multiple extrema were uncovered.

General matched, uniformly valid, leading order approximations for the damped and undamped cases were determined for a general forcing. These expressions were successfully compared to numerical results for a solution with given initial conditions. Applying the analysis to solutions for which the cnoidal function is a solution within a dispersive layer would further re-inforce the preceding results.

Examination of the parametric continuation of a general forcing extended the understanding of the behaviour of the solutions to the fKdV equation. The phenomenon of peak overcrowding was displayed when parametric continuation was applied to a problem with a forcing which yielded multiple peaks. The analysis of overcrowding was done at a fairly qualitative level. The least squares approximation supported the supposition relating peak width and critical values of overcrowding, but further quantitative analysis of this aspect may yield a stronger understanding.

Parametric continuation was difficult to employ for the second special case, 
when a forcing yielded a non-dispersive solution with multiple global minima. This thesis suggested the possible behaviour of such a forcing through variation of the detuning parameter by displaying key components of the behaviour. The analytic study of the peak formation and movement indicated that peaks would form at all of the global minima of the non-dispersive solution, and the peak pattern diagram did yield results showing that peaks formed at both minima, a phenomenon not seen in the other cases.

Global aspects arising from interactions between layers, such as peak shifting, and peak formation in multiple global minima cases require a different mathematical analysis. A layer based approach used in this thesis will not work and would need to be extended.

Stability was continued from previous work to fit a non-dispersive solution with multiple maxima and minima. The point of change in stability was analytically determined for the first peak of a given solution, but due to the limitations of the leading order approximations, subsequent points of change in stability could only be determined numerically. The examination of stability did yield a qualitatively identical bifurcation diagram to the case of a single mode forcing, which supported the underlying universality of the forced KdV equation.

Moreover, all of the aspects examined in this thesis upheld and extended the already known global behaviour of the forced KdV equation with a forcing of single mode to general forcing. 


\section{References}

[1] ABRAMOWITZ, M., STEGUN, I.A. 1965 Handbook of mathematical functions.

[2] ABLOWITZ, M.J., CLARKSON,P.A. 1991 Solitons, nonlinear evolution equations and inverse scattering.

[3] AMUNDSEN, D.E., COX, E.A., MORTELL, M.P., RECK, S. 2001 Evolution of nonlinear sloshing in a tank near half fundamental resonance.

[4] AMUNDSEN, D.E., COX, E.A., MORTELL, M.P. submitted to Zeitschrift fur Angewandte Mathematik und Physik (ZAMP) 2005 Asymptotic solutions for resonant sloshing of shallow water in a tank.

[5] CHESTER, W. 1968 Resonant oscillations of water-waves I. Theory.

[6] CHESTER, W., BONES, J.A. 1968 Resonant oscillations of water-waves II. Experiment.

[7] COX, E.A., MORTELL, M.P. 1983 The evolution of resonant oscillations in closed tubes.

[8] COX, E.A., MORTELL, M.P. 1986 The evolution of resonant oscillations.

[9] DIAS, F., VANDEN-BROECK, J.M. 2002 Steady two-layer flows over an obstacle

[10] DOEDEL E.J. 1981 AUTO: A program for the automatic bifurcation analysis of autonomous systems. 
REFERENCES

[11] GRADSHTEYN,I.S., RYZHIK, I.M. 1994 Table of integrals, series, and products.

[12] GRIMSHAW, R., YI, Z. 1993 Resonant generation of finite-amplitude waves by flow past topography on a beta-plane

[13] KEVORKIAN,J., COLE, J.D. 1996 Multiple scale and singular perturbation.

[14] KORTEWEG, D.J., de VRIES, G. 1895 On the change of form of long waves advancing in a rectangular canal, and on a new type of long stationary wave

[15] MALEEWONG, M., ASAVANANT, J., GRIMSHAW, R. 2005 Free surface flow under gravity and surface tension due to an applied pressure distribution

[16] MALKOV, M.A. 1996 Spatial chaos in weakly dispersive and viscous media: A nonperturbative theory of the driven Kdv-Burgers equation.

[17] MIURA, R.M., GARDNER, C.S., KRUSKAL, M.D. 1968 Korteweg-de Vries equations and generalizations. II. Existence of conservation laws and constants of motion

[18] OCKENDON, J.R., OCKENDON,H. 1973 Resonant surface-waves.

[19] OCKENDON,H., OCKENDON,J.R., JOHNSON, A.D. 1986 Resonant sloshing in shallow water. 